\title{
Interface Control Domain Decomposition (ICDD) Methods for Heterogeneous Problems
}

\author{
Marco Discacciati ${ }^{1}$, Paola Gervasio ${ }^{2 *}$ and Alfio Quarteroni ${ }^{3}$ \\ ${ }^{1}$ Laboratori de Càlcul Numèric (LaCàN), Departament de Matemàtica Aplicada III (MA3), Universitat Politècnica de \\ Catalunya (UPC BarcelonaTech), Campus Nord UPC - C2, E-08034 Barcelona, Spain. \\ 2 DICATAM, Università degli Studi di Brescia, via Branze 38, I-25123 Brescia, Italy. \\ ${ }^{3}$ Chair of Modelling and Scientific Computing, CMCS-MATHICSE, Ecole Polytechnique Fédérale de Lausanne, \\ Station 8, CH-1015 Lausanne, Switzerland and MOX, Politecnico di Milano, P.zza Leonardo da Vinci 32, I-20133 \\ Milano, Italy (on leave)
}

\begin{abstract}
SUMMARY
This paper is concerned with the solution of heterogeneous problems by the ICDD (Interface Control Domain Decomposition) method, a strategy introduced for the solution of partial differential equations (PDEs) in computational domains partitioned into subdomains that overlap. After reformulating the original boundary value problem by introducing new additional control variables, the unknown traces of the solution at internal subdomain interfaces, the latter are determined by requiring that the (a-priori) independent solutions in each subdomain undergo the minimization of a suitable cost functional.

We provide an abstract formulation for coupled heterogeneous problems and a general theorem of wellposedness for the associated ICDD problem. Then, we illustrate and validate an efficient algorithm based on the solution of the Schur-complement system restricted solely to the interface control variables by considering two kinds of heterogeneous boundary value problems: the coupling between pure advection and advection-diffusion equations, and the coupling between Stokes and Darcy equations. In the latter case we also compare the ICDD method with a classical approach based on the Beavers-Joseph-Saffman conditions. Copyright (c) 2013 John Wiley \& Sons, Ltd.
\end{abstract}

Received . .

KEY WORDS: Heterogeneous Problems, Domain Decomposition Methods, ICDD method, AdvectionDiffusion, Stokes-Darcy coupling, $h p$-Finite Elements

\section{INTRODUCTION}

Interface Control Domain Decomposition (ICDD) is a strategy for the solution of partial differential equations (PDEs) in computational domains partitioned into subdomains that overlap. It shares analogies and differences with similar strategies, most remarkably with those based on the Schwarz overlapping method (see $[1,2,3])$.

The distinguishing (and original) feature of ICDD method is that the original boundary value problem is reformulated with the help of new additional variables, the unknown traces of the solution at internal subdomain interfaces, that play the role of control variables. Their determination is made possible by the requirement that the (a-priori) independent solutions in each subdomain undergo the minimization of a suitable cost functional.

What distinguishes between different kinds of ICDD methods is the role (and physical meaning) of the interface control variables - indeed, they can represent either Dirichlet, Neumann, or Robin

\footnotetext{
${ }^{*}$ Correspondence to: Paola Gervasio, DICATAM, Università degli Studi di Brescia, via Branze 38, I-25123 Brescia, Italy.

E-mail: paola.gervasio@unibs.it 
traces of the subdomain unknowns - and the type of cost functional chosen - typically it expresses the gap between the two solutions in overlapping areas or on internal interfaces in different norms.

When the ICDD method is applied to classical homogeneous PDEs, as done e.g. in $[4,5]$ for elliptic and Stokes equations, it can be regarded as (yet) another domain decomposition (DD) method.

However, ICDD methods become especially attractive when applied to solve heterogeneous PDEs, that is to couple problems where different kinds of PDEs are set up in different subdomains. A noticeable case occurs in multiphysics applications, where the different PDEs are designed to model different kinds of physics. Examples include fluid-structure interactions (modeled e.g. by NavierStokes equations coupled with the system of linear or nonlinear elasticity), the coupling between surface and subsurface flows (modeled e.g. by the Stokes and Darcy equations [6, 7]), etc. In those cases, the minimization problem set on the interface control variables that is enforced by ICDD methods can in principle assure the correct matching between the two different physics, without requiring the a-priori determination of interface transmission conditions across a sharp interface.

In this context, ICDD methods can be regarded not only as a new way to numerically solve a given boundary value problem, but also as a new (alternative) way to model and simulate efficiently multiphysics problems.

In this paper we first focus our attention on the coupling of an advection-diffusion equation with an advection equation, the latter being derived from the former by dropping the diffusion term in a subregion of the computational domain. The problem is simple but not too simple for testing the properties of the ICDD method.

After replacing the minimization problem by its discrete version obtained using the $h p$-Galerkin approximation, we analyze the well-posedness of the discrete ICDD method in an abstract heterogeneous framework and illustrate the corresponding solution algorithm. Then, we present numerical results for the coupling between advection-diffusion and advection equations, showing that the ICDD method enjoys excellent convergence properties and represents a novel and fairly general paradigm to face heterogeneous PDEs in domain decomposition environments.

In the second part of the paper we consider the ICDD method to couple Stokes and Darcy equations. The method was formerly proposed and analyzed in [8]. Here we further investigate it in some new applications, by considering non-homogenous porous media, non-straight interfaces and "near normal flows" [9] (the less investigated cases in literature), and we compare it with the sharp interface approach based on the classical Beavers-Joseph-Saffman (BJS) conditions [10, 11]. Numerical results show that the ICDD method turns out to be a valid and competitive alternative to the Sharp Interface approach with BJS condition (SI-BJS in brief) especially for what concerns the accuracy of the solution, the easiness of implementation and the associated computational cost.

The outline of the paper is as follows. In Section 2 we present the ICDD method in the case of the heterogeneous coupling between Advection and Advection-Diffusion (A-AD) problems and in Section 3 we introduce its discretization. In Section 4 we extend the ICDD method to the StokesDarcy problem, while in Section 5 we introduce an abstract framework for heterogeneous couplings, define the associated optimality systems, reformulate the ICDD method algebraically and prove the well-posedeness of the discrete minimization problem.

Finally, Section 6 is devoted to the analysis of the numerical results for the A-AD coupling, and Section 7 presents some test cases for the Stokes-Darcy coupling.

\section{ICDD FOR THE COUPLING OF ADVECTION WITH ADVECTION/DIFFUSION PROBLEMS}

\subsection{Problem setting}

Let $\Omega \subset \mathbb{R}^{d}(d=1,2,3)$ be an open bounded domain with boundary $\partial \Omega, \partial \Omega_{D}$ and $\partial \Omega_{N}$ two open subsets of $\partial \Omega$ such that $\partial \Omega=\overline{\partial \Omega_{D} \cup \partial \Omega_{N}}$ and $\partial \Omega_{D} \cap \partial \Omega_{N}=\emptyset$. Let $L_{2}$ be the second order linear elliptic operator

$$
L_{2} u=\operatorname{div}(-\nu \nabla u+\mathbf{b} u)+\gamma u,
$$



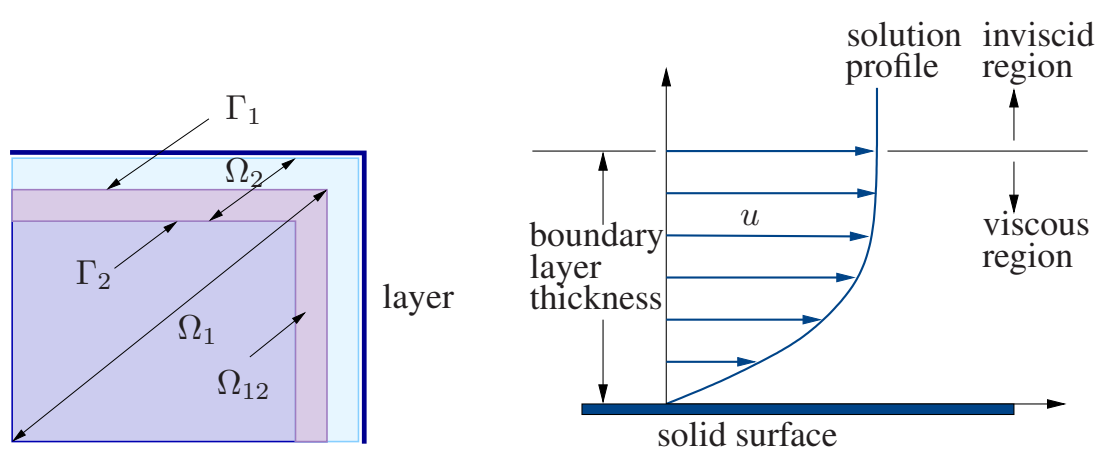

Figure 1. On the left, graphic representation of a 2D A-AD heterogeneous coupling. On the right, the sketch of a boundary layer

where $\nu, \mathbf{b}$, and $\gamma$ are suitable functions we specify better in the next subsection.

Let us consider the boundary-value problem

Problem P:

$$
\begin{array}{ll}
L_{2} u=f & \text { in } \Omega \\
u=\phi_{D} & \text { on } \partial \Omega_{D} \\
\partial_{n_{L_{2}}} u=\phi_{N} & \text { on } \partial \Omega_{N},
\end{array}
$$

where $f, \phi_{D}$, and $\phi_{N}$ are assigned functions satisfying suitable compatibility conditions on $\overline{\partial \Omega_{N}} \cap \overline{\partial \Omega_{D}}$ (see [12]), and $\partial_{n_{L_{2}}} u$ denotes the conormal derivative of $u$ :

$$
\partial_{n_{L_{2}}} u=\nu \mathbf{n} \cdot \nabla u-\mathbf{b} \cdot \mathbf{n} u
$$

$\mathbf{n}$ being the unit normal vector external to $\partial \Omega$.

When advection dominates diffusion, boundary and/or internal layer may occur (see Fig. 1). In such case the viscous term is essential in the region close to the layer, but it may be neglected far from the layer. Dropping the viscous term in the latter region yields a reduced order differential operator therein.

For this reason, we split $\Omega$ into 2 overlapping subdomains $\Omega_{1}$ and $\Omega_{2}$ such that $\bar{\Omega}=\overline{\Omega_{1} \cup \Omega_{2}}$, we set $\Omega_{12}=\Omega_{1} \cap \Omega_{2}, \Gamma_{i}=\partial \Omega_{i} \backslash \partial \Omega$, for $i=1,2$ (see Fig. 1 for a simple example in $\mathbb{R}^{2}$ ) and we look for two functions $u_{1}$ and $u_{2}$ (defined in $\bar{\Omega}_{1}$ and $\bar{\Omega}_{2}$, respectively) such that $u_{1}$ satisfies the advection equation

$$
L_{1} u_{1}=\operatorname{div}\left(\mathbf{b} u_{1}\right)+\gamma u_{1}=f \quad \text { in } \Omega_{1},
$$

while $u_{2}$ satisfies the advection-diffusion equation

$$
L_{2} u_{2}=f \quad \text { in } \Omega_{2} .
$$

For any non-empty subset $\Gamma \subseteq \partial \Omega_{1}$, we define

$$
\begin{array}{ll}
\text { the inflow part of } \Gamma: & \Gamma^{\text {in }}=\{\mathbf{x} \in \Gamma: \mathbf{b}(\mathbf{x}) \cdot \mathbf{n}(\mathbf{x})<0\}, \\
\text { the outflow part of } \Gamma: & \Gamma^{\text {out }}=\{\mathbf{x} \in \Gamma: \mathbf{b}(\mathbf{x}) \cdot \mathbf{n}(\mathbf{x})>0\}
\end{array}
$$

and we set $\Gamma^{0}=\Gamma \backslash\left(\overline{\Gamma^{i n}} \cup \overline{\Gamma^{o u t}}\right)$.

Boundary conditions on the external boundaries $\partial \Omega_{i} \backslash \Gamma_{i}$ are inherited from the original problem (2), taking care that the first-order problem is well posed when the Dirichlet condition is assigned only on the inflow boundary $\left(\partial \Omega_{1}\right)^{i n}$, thus $\partial \Omega_{D}$ is such that $\left(\partial \Omega_{1} \backslash \Gamma_{1}\right)^{i n} \subset \partial \Omega_{D}$.

It remains to set boundary conditions on the internal boundaries $\Gamma_{1}^{i n}$ and $\Gamma_{2}$ (that from now on we call interfaces). For the sake of simplicity, we put $\partial \Omega_{N}=\emptyset$ (thus $\partial \Omega_{D}=\partial \Omega$ ) and $\phi_{D}=0$.

We set $\Gamma_{2}^{n z}=\Gamma_{2} \backslash \Gamma_{2}^{0}$ and we introduce two unknown functions $\lambda_{1}$ and $\lambda_{2}$ defined on the interfaces $\Gamma_{1}^{i n}$ and $\Gamma_{2}^{n z}$, respectively, that are named controls and play the role of Dirichlet data for the following Advection and Advection-Diffusion subproblems, respectively: 
Advection problem:

$$
\begin{array}{ll}
L_{1} u_{1}=f_{1} & \text { in } \Omega_{1} \\
u_{1}=\lambda_{1} & \text { on } \Gamma_{1}^{i n} \\
& \\
u_{1}=0 & \text { on }\left(\partial \Omega_{1} \backslash \Gamma_{1}\right)^{i n}
\end{array}
$$

Advection-Diffusion system:

$$
\begin{array}{ll}
L_{2} u_{2}=f_{2} & \text { in } \Omega_{2} \\
u_{2}=\lambda_{2} & \text { on } \Gamma_{2}^{n z} \\
\nu \frac{\partial u_{2}}{\partial n_{2}}=0 & \text { on } \Gamma_{2}^{0} \\
u_{2}=0 & \text { on } \partial \Omega_{2} \backslash \Gamma_{2} .
\end{array}
$$

The unknown controls $\lambda_{1}$ and $\lambda_{2}$ can be found by minimizing a suitable cost functional that measures the gap between the solutions $u_{1}$ and $u_{2}$ on the interfaces $\Gamma_{1}^{i n}$ and $\Gamma_{2}$ in a suitable norm, i.e.

$$
\left(\lambda_{1}, \lambda_{2}\right)=\underset{\left(\mu_{1}, \mu_{2}\right)}{\operatorname{argmin}}\left[J\left(\mu_{1}, \mu_{2}\right)=\frac{1}{2}\left\|u_{1}\left(\mu_{1}\right)-u_{2}\left(\mu_{2}\right)\right\|_{\Gamma_{1}^{i n} \cup \Gamma_{2}^{n z}}^{2}\right] .
$$

This expression is a formal one. We will better precise the norm used in (6) after the definition of the functional spaces in which both solutions and controls make sense.

The minimization problem (6) with constraints (5) is in fact an optimal control problem and it can be analyzed by using the classical Optimal Control (OC) theory (see, e.g., [13]). According to the OC terminology, the controls are of boundary type (actually they are interface controls) and the observation is of boundary type.

\section{Remark 2.1}

The idea of using virtual controls at the interfaces to solve homogeneous elliptic problems via overlapping domain decomposition appears in the papers [14, 15], where, however, the authors choose cost functionals measuring the gap between $u_{1}$ and $u_{2}$ on the whole overlap $\Omega_{12}$, as e.g.

$$
J_{\Omega_{12}}\left(\mu_{1}, \mu_{2}\right)=\frac{1}{2}\left\|u_{1}\left(\mu_{1}\right)-u_{2}\left(\mu_{2}\right)\right\|_{L^{2}\left(\Omega_{12}\right)}^{2} .
$$

According to the classical terminology of $\mathrm{OC}$ problems, this is a case of distributed observation.

Interface observations like in (6) were formerly introduced in [4] in the context of homogeneous elliptic problems. Numerical results presented in [4] show that interface observation yields iterative domain decomposition methods that are more efficient and cheaper than those arising from distributed observation, even if the optimality system associated with the former approach is not symmetric.

\section{Remark 2.2}

A preliminary study of the A-AD coupling with overlapping subdomains has been carried out in $[16,17,18]$ but with a cost functional as in (7).

From the theoretical point of view, we notice that minimizing $\left\|u_{1}-u_{2}\right\|_{L^{2}\left(\Omega_{12}\right)}^{2}$ is meaningful for homogeneous couplings, i.e. when the same differential operator is considered in both subdomains, for which both $u_{1}$ and $u_{2}$ represent the (common) restriction of the original solution in the overlap. On the contrary, in the heterogeneous case, it is not necessarily true that the two solutions must coincide on the whole overlap (and in general they do not).

Moreover, efficiency considerations like those outlined in the previous remark led us to replace (7) with (6).

\subsection{Functional spaces and weak formulation of the ICDD problem}

To continue we need to introduce some assumptions and useful spaces.

Let $\nu \in L^{\infty}(\Omega)$ be such that $\exists \underline{\nu}>0: \nu(\mathbf{x}) \geq \underline{\nu} \forall \mathbf{x} \in \Omega, \mathbf{b} \in\left[W^{1, \infty}(\Omega)\right]^{d}$ and $\gamma \in L^{\infty}(\Omega)$ with $\gamma(\mathbf{x}) \geq 0$ in $\Omega$. We assume that these functions are such that the elliptic operator is coercive (see [4] for a detailed description of the required assumptions). Moreover, $f \in L^{2}(\Omega), \phi_{D} \in$ $H^{1 / 2}\left(\partial \Omega_{D}\right), \phi_{N} \in H^{-1 / 2}\left(\partial \Omega_{N}\right)$ are assigned functions satisfying suitable compatibility conditions on $\overline{\partial \Omega_{N}} \cap \overline{\partial \Omega_{D}}$. 
Given an open domain $D \subset \mathbb{R}^{d}$ with Lipschitz boundary $\partial D$, and given $S \subseteq \partial D$, we define

$$
L_{\mathbf{b}}^{2}(S)=\left\{v: S \rightarrow \mathbb{R}: v \sqrt{\left|\mathbf{b} \cdot \mathbf{n}_{S}\right|} \in L^{2}(S)\right\},
$$

where $\mathbf{n}_{S}$ denotes the outward normal versor to $D$ on $S$, and

$$
X_{\mathbf{b}}(D)=\left\{v \in L^{2}(D), \operatorname{div}(\mathbf{b} v) \in L^{2}(D): v \in L_{\mathbf{b}}^{2}(\partial D)\right\} .
$$

They are both Hilbert spaces (see [19]) with respect to their natural norms:

$$
\|u\|_{L_{\mathbf{b}}^{2}(S)}=\left(\int_{S}|\mathbf{b} \cdot \mathbf{n}| u^{2} d S\right)^{1 / 2},\|u\|_{X_{\mathbf{b}}(D)}=\left(\|u\|_{L^{2}(D)}^{2}+\|\operatorname{div}(\mathbf{b} u)\|_{L^{2}(D)}^{2}+\|u\|_{L_{\mathbf{b}}^{2}(\partial D)}^{2}\right)^{1 / 2} .
$$

Then, we define the spaces of the controls:

$$
\Lambda_{1}=L_{\mathbf{b}}^{2}\left(\Gamma_{1}^{i n}\right), \quad \Lambda_{2}=H_{00}^{1 / 2}\left(\Gamma_{2}^{n z}\right),
$$

the following Hilbert spaces:

$$
\begin{array}{cl}
V_{1}=\left\{v_{1} \in X_{\mathbf{b}}\left(\Omega_{1}\right): v_{1}=0 \text { on }\left(\partial \Omega_{1} \backslash \Gamma_{1}\right)^{i n}\right\}, & V_{1}^{0}=\left\{v_{1} \in X_{\mathbf{b}}\left(\Omega_{1}\right): v_{1}=0 \text { on }\left(\partial \Omega_{1}\right)^{i n}\right\}, \\
V_{2}=\left\{v_{2} \in H^{1}\left(\Omega_{2}\right): v_{2}=0 \text { on } \partial \Omega_{2} \backslash \Gamma_{2}\right\}, & V_{2}^{0}=\left\{v_{2} \in H^{1}\left(\Omega_{2}\right): v_{2}=0 \text { on } \partial \Omega_{2} \backslash \Gamma_{2}^{0}\right\},
\end{array}
$$

and we set $\mathbf{V}=V_{1} \times V_{2}, \mathbf{V}^{0}=V_{1}^{0} \times V_{2}^{0}$ and $\mathbf{\Lambda}=\Lambda_{1} \times \Lambda_{2}$.

Finally we define the bilinear forms:

$$
\begin{array}{ll}
a_{1}: V_{1} \times V_{1} \rightarrow \mathbb{R} \quad a_{1}\left(u_{1}, v_{1}\right)=-\int_{\Omega_{1}} u_{1} \mathbf{b} \cdot \nabla v_{1}+\int_{\Omega_{1}} \gamma u_{1} v_{1}+\int_{\partial \Omega_{1}^{\text {out }}} \mathbf{b} u_{1} v_{1}, \\
a_{2}: V_{2} \times V_{2} \rightarrow \mathbb{R} \quad a_{2}\left(u_{2}, v_{2}\right)=\int_{\Omega_{2}}\left(\nu \nabla u_{2}-\mathbf{b} u_{2}\right) \cdot \nabla v_{2}+\int_{\Omega_{2}} \gamma u_{2} v_{2},
\end{array}
$$

the linear functionals $F_{i}: V_{i} \rightarrow \mathbb{R}$

$$
F_{i}\left(v_{i}\right)=\int_{\Omega_{i}} f v_{i}
$$

and the extension operators

$$
\begin{aligned}
& E_{1}: \Lambda_{1} \rightarrow V_{1}, \quad E_{1} \lambda_{1}=\lambda_{1} \text { on } \Gamma_{1}^{i n} \\
& E_{2}: \Lambda_{2} \rightarrow V_{2}, \quad E_{2} \lambda_{2}=\lambda_{2} \text { on } \Gamma_{2}^{n z} .
\end{aligned}
$$

The weak form of system (3), (4), (6) reads as follows. Given $f \in L^{2}(\Omega)$, we look for $u_{1,0} \in V_{1}^{0}$, $u_{2,0} \in V_{2}^{0}, \lambda_{1} \in \Lambda_{1}, \lambda_{2} \in \Lambda_{2}$ such that

$$
\begin{array}{r}
a_{1}\left(u_{1,0}, v_{1}\right)+a_{1}\left(E_{1} \lambda_{1}, v_{1}\right)=\mathcal{F}_{1}\left(v_{1}\right) \quad \forall v_{1} \in V_{1}^{0} \\
a_{2}\left(u_{2,0}, v_{2}\right)+a_{2}\left(E_{2} \lambda_{2}, v_{2}\right)=\mathcal{F}_{2}\left(v_{2}\right) \quad \forall v_{2} \in V_{2}^{0} \\
u_{1}\left(\mu_{1}\right)=u_{1,0}+E_{1} \mu_{1}, \quad u_{2}\left(\mu_{2}\right)=u_{2,0}+E_{2} \mu_{2}, \\
\left(\lambda_{1}, \lambda_{2}\right)=\underset{\left(\mu_{1}, \mu_{2}\right)}{\operatorname{argmin}}\left[J\left(\mu_{1}, \mu_{2}\right)=\frac{1}{2}\left\|u_{1}\left(\mu_{1}\right)-u_{2}\left(\mu_{2}\right)\right\|_{L_{\mathbf{b}}^{2}\left(\Gamma_{1}^{i n} \cup \Gamma_{2}^{n z}\right)}^{2 n z}\right] .
\end{array}
$$

Under the assumptions introduced for $\nu$ and $\gamma$, if moreover $\exists \beta_{0}>0$ such that $\frac{1}{2} \nabla \cdot \mathbf{b}+\gamma \geq \beta_{0}$ in $\Omega_{1}$, and under the assumption that $\lambda_{i} \in \Lambda_{i}$ (for $i=1,2$ ) are given, both problems (10) and (11) are well-posed (see. e.g. [19, 20]).

In the next Section we write the discrete counterpart of (10)-(13) and prove its well-posedness. 


\section{Remark 2.3}

When the computational domain is partitioned into two non-overlapping subdomains $\Omega_{1}, \Omega_{2}$ with sharp interface (i.e. such that $\bar{\Omega}_{1} \cup \bar{\Omega}_{2}=\bar{\Omega}, \Omega_{1} \cap \Omega_{2}=\emptyset$ and $\Gamma=\partial \Omega_{1} \cap \partial \Omega_{2}$ ), the heterogeneous A-AD coupling has been analyzed in $[19,20,18,21]$. In $[19,21]$ the authors provided a suitable set of interface conditions on the sharp interface $\Gamma$ of the decomposition, i.e.

$$
\begin{array}{ll}
u_{1}=u_{2} & \text { on } \Gamma^{\text {in }} \\
\mathbf{b} \cdot \mathbf{n} u_{1}+\nu \mathbf{n} \cdot \nabla u_{2}-\mathbf{b} \cdot \mathbf{n} u_{2}=0 & \text { on } \Gamma
\end{array}
$$

where $\mathbf{n}$ is the unit normal vector to $\Gamma$ oriented from $\Omega_{1}$ to $\Omega_{2}$.

These conditions express the continuity of the velocity field across the inflow part of the unique interface $\Gamma$ and the continuity of the fluxes across the whole interface $\Gamma$. In such case a suitable version of the classical Dirichlet-Neumann method $([22,19])$ can be successfully applied to solve the heterogeneous problem.

Other domain decomposition approaches based on virtual controls or extended variational formulation on non-overlapping subdomains have been addressed in [18, 20, 23] to face similar heterogeneous coupled problems.

\section{3. $h p$-FEM DISCRETIZATION}

For $i=1,2$, let $\mathcal{T}_{i}$ be a partition of the computational domain $\Omega_{i} \subset \mathbb{R}^{d}$ in either simplices or quadrilaterals/hexahedra (quadrilaterals when $d=2$ and hexahedra when $d=3$ ). We denote by $\hat{T}$ the reference element, that can be either the reference simplex with vertexes 0 and the points on the axis whose distance from the origin is 1 , or the $d$-dimensional cube $(-1,1)^{d}$.

We name simplicial the partitions composed of simplices and quad those partitions formed by quadrilaterals/hexahedra. The first ones are typical of classical FEM, the others of Spectral Elements Methods (SEM) with tensorial structure (see [24, 25]). From now on we group them under $h p-$ FEM.

We suppose that each element $T \in \mathcal{T}_{i}$ is obtained by a $C^{1}$ diffeomorphism $\mathbf{F}_{T}$ of the reference element $\hat{T}$ and we suppose that two adjacent elements of $\mathcal{T}_{i}$ share a common vertex, a complete edge, or a complete face (when $d=3$ ). For each $T \in \mathcal{T}_{i}$ we denote by $h_{T}=\operatorname{diam}(T)=\max _{\mathbf{x}, \mathbf{y} \in T}|\mathbf{x}-\mathbf{y}|$ the diameter of the element $T$ and we define $h_{i}=\max _{T \in \mathcal{T}_{i}} h_{T}$. Then, when simplicial partitions are considered, we require that the grid is regular in each $\Omega_{i}$ (see, e.g., [26]).

We assume that the meshes $\mathcal{T}_{1}$ and $\mathcal{T}_{2}$ match on $\bar{\Omega}_{12}$ so that the interfaces $\Gamma_{1}$ and $\Gamma_{2}$ do not cut any element of the overlapping subdomains $\Omega_{2}$ and $\Omega_{1}$, respectively.

Given an integer $p \geq 1$, let us denote by $\mathbb{P}_{p}$ the space of polynomials whose global degree is less than or equal to $p$ in the variables $x_{1}, \ldots, x_{d}$ and by $\mathbb{Q}_{p}$ the space of polynomials that are of degree less than or equal to $p$ with respect to each variable $x_{1}, \ldots, x_{d}$. The space $\mathbb{P}_{p}$ is associated with simplicial partitions, while $\mathbb{Q}_{p}$ with quad ones. We introduce the following finite dimensional spaces in $\bar{\Omega}_{i}$ :

$$
X_{i, h}^{p}=\left\{v \in C^{0}\left(\bar{\Omega}_{i}\right): v_{\mid T} \in \mathcal{Q}_{p}, \forall T \in \mathcal{T}_{i}\right\}
$$

where $\mathcal{Q}_{p}=\mathbb{P}_{p}$ in the simplicial case and $\mathcal{Q}_{p}=\mathbb{Q}_{p} \circ \mathbf{F}_{T}^{-1}$ for quads.

Finally, let $\mathcal{M}_{i}$ be the set of the nodes $\mathbf{x}_{j}$ of the mesh $\mathcal{T}_{i}$.

Then, the finite dimensional spaces in which we look for the $h p$-FEM solution are, for $i=1,2$,

$$
V_{i, h}=V_{i} \cap X_{i, h}^{p}, \quad V_{i, h}^{0}=V_{i}^{0} \cap X_{i, h}^{p}
$$

for a suitable polynomial degree $p$, while the spaces of discrete Dirichlet controls are defined as

$$
\begin{aligned}
& \Lambda_{1, h}=\left\{\lambda_{1, h} \in C^{0}\left(\overline{\Gamma_{1}^{i n}}\right): \exists v_{1, h} \in V_{1, h} \text { with } \lambda_{1, h}=\left.v_{1, h}\right|_{\Gamma_{1}^{i n}}\right\}, \\
& \Lambda_{2, h}=\left\{\lambda_{2, h} \in C^{0}\left(\overline{\Gamma_{2}^{n z}}\right): \exists v_{2, h} \in V_{2, h} \text { with } \lambda_{2, h}=\left.v_{2, h}\right|_{\Gamma_{2}^{n z}} ^{n z} .\right.
\end{aligned}
$$


We denote by $N_{\Omega_{1}}, N_{\Gamma_{1}}, N_{\Omega_{2}}$, and $N_{\Gamma_{2}}$ the cardinality of $V_{1, h}^{0}, \Lambda_{1, h}, V_{2, h}^{0}$, and $\Lambda_{2, h}$, respectively.

In each discrete functional space we consider the basis of the characteristic Lagrange polynomials associated with the nodes of $\mathcal{M}_{i}$ and we denote by $\varphi_{i, \ell}$ (for $i=1,2$ and $\ell=1, \ldots, N_{\Omega_{i}}$ ) the basis functions of $V_{1, h}^{0}$, and $V_{2, h}^{0}$, respectively.

The basis functions in $\Lambda_{1, h}$ are denoted by $\eta_{1, \ell}$ (for $\ell=1, \ldots, N_{\Gamma_{1}}$ ) and they are defined by restricting to $\Gamma_{1}^{i n}$ the basis functions of $V_{1, h}$ that are not identically null on $\Gamma_{1}^{i n}$. Similarly we define the basis function $\eta_{2, \ell}$ (for $\ell=1, \ldots, N_{\Gamma_{2}}$ ) of $\Lambda_{2, h}$, starting from the basis in $V_{2, h}$.

Because of the difficulty of computing integrals exactly for large $p$, typically when quad partitions are adopted, Legendre-Gauss-Lobatto quadrature formulas are used to approximate both the bilinear forms $a_{i}$ and the $L^{2}$-inner products in $\Omega_{i}$ (as well as on the interfaces). This leads to the so-called Galerkin approach with Numerical Integration (G-NI) [24, 27] and to the Spectral Element Method with Numerical Integration (SEM-NI). For this reason we replace $a_{i}$ with $a_{i, h}$ and $\mathcal{F}_{i}$ with $\mathcal{F}_{i, h}$, understanding that when exact quadrature formulas are used, then $a_{i, h}=a_{i}$ and $\mathcal{F}_{i, h}=\mathcal{F}_{i}$.

We define the discrete counterpart of the extension operators:

$$
\begin{array}{ll}
E_{1, h}: \Lambda_{1, h} \rightarrow V_{1, h}, \quad & E_{1, h} \lambda_{1, h}=\lambda_{1, h} \text { on } \Gamma_{1}^{i n}, \\
& E_{1, h} \lambda_{1, h}\left(\mathbf{x}_{j}\right)=0 \text { for any } \mathbf{x}_{j} \in \mathcal{M}_{1} \cap\left(\bar{\Omega}_{1} \backslash \Gamma_{1}^{i n}\right), \\
E_{2, h}: \Lambda_{2, h} \rightarrow V_{2, h}, \quad & E_{2, h} \lambda_{2, h}=\lambda_{2, h} \text { on } \Gamma_{2}^{n z}, \\
& E_{2, h} \lambda_{2, h}\left(\mathbf{x}_{j}\right)=0 \text { for any } \mathbf{x}_{j} \in \mathcal{M}_{2} \cap\left(\bar{\Omega}_{2} \backslash \Gamma_{2}^{n z}\right) .
\end{array}
$$

Extension operators might also be of non-interpolatory type, depending upon the kind of governing PDEs or the space discretization approach.

The discrete counterpart of (10)-(13) reads: given $f \in L^{2}(\Omega)$, we look for $u_{1,0, h} \in V_{1, h}^{0}, u_{2,0, h} \in$ $V_{2, h}^{0}, \lambda_{1, h} \in \Lambda_{1, h}$, and $\lambda_{2, h} \in \Lambda_{2, h}$ such that

$$
\begin{array}{rr}
a_{1, h}\left(u_{1,0, h}, v_{1, h}\right)+a_{1, h}\left(E_{1, h} \lambda_{1, h}, v_{1, h}\right)=\mathcal{F}_{1, h}\left(v_{1, h}\right) & \forall v_{1, h} \in V_{1, h}^{0}, \\
a_{2, h}\left(u_{2,0, h}, v_{2, h}\right)+a_{2, h}\left(E_{2, h} \lambda_{2, h}, v_{2, h}\right)=\mathcal{F}_{2, h}\left(v_{2, h}\right) & \forall v_{2, h} \in V_{2, h}^{0}, \\
u_{1, h}\left(\lambda_{1, h}\right)=u_{1,0, h}+E_{1, h} \lambda_{1, h}, \quad u_{2, h}\left(\lambda_{2, h}\right)=u_{2,0, h}+E_{2, h} \lambda_{2, h}, \\
\left(\lambda_{1, h}, \lambda_{2, h}\right)=\underset{\left(\mu_{1, h}, \mu_{2, h}\right)}{\operatorname{argmin}}\left[J\left(\mu_{1, h}, \mu_{2, h}\right)=\frac{1}{2}\left\|u_{1, h}\left(\mu_{1, h}\right)-u_{2, h}\left(\mu_{2, h}\right)\right\|_{L_{\mathbf{b}}^{2}\left(\Gamma_{1}^{i n} \cup \Gamma_{2}^{n z}\right)}^{2}\right] .
\end{array}
$$

\section{ICDD FOR THE STOKES-DARCY COUPLING}

The ICDD strategy can be applied also to the heterogeneous coupled Stokes-Darcy system, used to model the filtration of free fluids through porous media. The physical setting of this problem is as follows. We consider an open bounded domain $\Omega \subset \mathbb{R}^{d}(d=2,3)$ split into two non-overlapping regions: $\Omega_{1}$ occupied by a confined incompressible fluid and $\Omega_{2}$ formed by a porous medium such that $\bar{\Omega}=\bar{\Omega}_{1} \cup \bar{\Omega}_{2}$ with $\Omega_{1} \cap \Omega_{2}=\emptyset$. We denote by $\Gamma=\partial \Omega_{1} \cap \partial \Omega_{2}$ the ideal hyper-surface separating the fluid and the porous region through which the fluid can move freely from one subdomain into the other (see Fig. 2, left). We assume that $\Omega$ has a Lipschitz continuous boundary $\partial \Omega$ and we also require that $\Gamma$ is regular enough to guarantee that both $\partial \Omega_{1}$ and $\partial \Omega_{2}$ are Lipschitz continuous too.

In the steady case when the Reynolds number in the fluid is not too large, the fluid-porous medium system can be modeled coupling the Stokes equations and Darcy's law (see, e.g., [6, 28, 18]).

More precisely, the fluid satisfies the Stokes equations in $\Omega_{1}$ :

$$
\begin{array}{ll}
-\nabla \cdot \mathbf{T}_{1}\left(\mathbf{u}_{1}, p_{1}\right)=\mathbf{f}_{1} & \text { in } \Omega_{1} \\
\nabla \cdot \mathbf{u}_{1}=0 & \text { in } \Omega_{1}
\end{array}
$$

where $\mathbf{u}_{1}$ and $p_{1}$ denote, respectively, the velocity and the pressure of the free fluid in $\Omega_{1}$. $\mathbf{T}_{1}\left(\mathbf{u}_{1}, p_{1}\right)=\mu\left(\nabla \mathbf{u}_{1}+\left(\nabla \mathbf{u}_{1}\right)^{T}\right)-p_{1} \mathbf{I}$ is the Cauchy stress tensor, where $\mu>0$ is the dynamic viscosity of the fluid. Finally, $\mathbf{f}_{1} \in\left[L^{2}\left(\Omega_{1}\right)\right]^{d}$ is a given external force. 

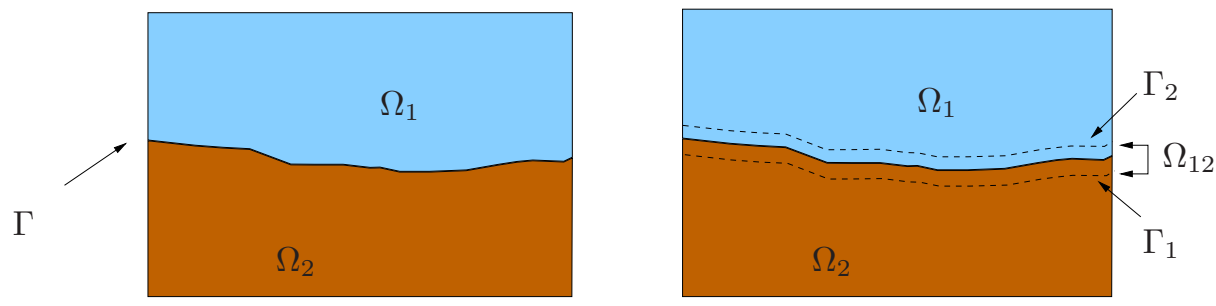

Figure 2. Representation of a 2D section of a possible computational domain for the coupled free/porousmedia flow problem. At left a decomposition with sharp interface $\Gamma$, at right a decomposition with overlap

In $\Omega_{2}$ the fluid obeys Darcy's law ([29]):

$$
\begin{array}{ll}
\boldsymbol{\alpha}^{-1} \mathbf{u}_{2}+\nabla p_{2}=\mathbf{f}_{2} & \text { in } \Omega_{2} \\
\nabla \cdot \mathbf{u}_{2}=0 & \text { in } \Omega_{2}
\end{array}
$$

where $\mathbf{u}_{2}$ is the Darcy velocity (specific discharge) and $p_{2}$ is the (average) pressure inside the porous medium. We have denoted $\alpha=\kappa / \mu$ where $\kappa$ is the symmetric and positive definite intrinsic permeability tensor. Finally, $\mathbf{f}_{2} \in L^{2}\left(\Omega_{2}\right)$ is an assigned external force (e.g., gravity).

In this coupled setting, adopting the Darcy model is physically significant either when the characteristic length scale of the pores is very small with respect to the characteristic length of the Stokes domain or when the Reynolds number in the Darcy domain is small with respect to 1.

Besides suitable boundary conditions on $\partial \Omega_{1} \backslash \Gamma$ and $\partial \Omega_{2} \backslash \Gamma$, transmission conditions must be imposed across the interface $\Gamma$ to represent the filtration process occurring therein. A commonly accepted set of interface conditions reads [28]:

$$
\begin{array}{ll}
\mathbf{u}_{1} \cdot \mathbf{n}=\mathbf{u}_{2} \cdot \mathbf{n} & \text { on } \Gamma \\
-\left(\mathbf{T}_{1}\left(\mathbf{u}_{1}, p_{1}\right) \mathbf{n}\right) \cdot \mathbf{n}=p_{2} & \text { on } \Gamma \\
-\left(\mathbf{T}_{1}\left(\mathbf{u}_{1}, p_{1}\right) \mathbf{n}\right) \cdot \boldsymbol{\tau}=\frac{\alpha_{B J} \mu}{\sqrt{\boldsymbol{\tau}^{T} \boldsymbol{\kappa} \boldsymbol{\tau}}} \mathbf{u}_{1} \cdot \boldsymbol{\tau} & \text { on } \Gamma
\end{array}
$$

where $\mathbf{n}$ is the normal unit vector to $\Gamma$ directed outwards from $\Omega_{1}, \tau$ represents a set of linear independent unit tangential vectors to $\Gamma$ with $\boldsymbol{\tau} \cdot \mathbf{n}=0$, and $\alpha_{B J}$ is a dimensionless coefficient depending on the geometrical characteristics of the porous medium.

Condition $(25)_{1}$ is a consequence of the conservation of mass across the interface and $(25)_{2}$ is due to the balance of normal forces (see, e.g., [30, 31, 32]). Equality $(25)_{3}$ is the so-called BeaversJoseph-Saffman (BJS) interface condition [10, 11, 33, 32, 30, 34] that establishes proportionality between the tangential component of the flux and that of the free velocity, but it is not a coupling condition.

The mathematical analysis of the steady Stokes/Darcy problem can be found in [28, 32]. In [28] the authors propose and analyze iterative substructuring domain decomposition methods of Dirichlet-Neumann and Robin-Robin type to efficiently solve the coupled problem.

Another possible coupling paradigm is to introduce a transition region with positive small thickness between the fluid region and the porous medium. In [35, 36], Ochoa-Tapia and Whitaker solve the Stokes equations in the free-fluid domain, the Stokes problem with an additional term featuring a variable porosity inside the transition region, and the Darcy model with Brinkman correction in the porous domain. In [8], the ICDD technique is proposed to couple the Stokes and the Darcy equations as an alternative approach to sharp interface with conditions (25) based on the use of a thin transition region. In this context, the transition region merely represents the overlap $\Omega_{12}=\Omega_{1} \cap \Omega_{2} \neq \emptyset$ of two subdomains, one $\left(\Omega_{1}\right)$ in which we solve the free fluid (Stokes) equations, the other $\left(\Omega_{2}\right)$ where we solve the porous medium (Darcy) equation. The coupling between the two flow regimes is not modeled but it is achieved by imposing in a least-squares sense the continuity of velocity and pressure across the interfaces $\Gamma_{i}=\partial \Omega_{i} \backslash \partial \Omega(i=1,2)$, see Figure 2, right. 
As for the A-AD case, the ICDD method for the Stokes-Darcy problem consists in introducing two unknown control functions $\lambda_{1}$ and $\lambda_{2}$ defined on the interfaces $\Gamma_{1}$ and $\Gamma_{2}$, respectively, that play the role of Dirichlet data for the following Stokes and Darcy subproblems:

$$
\begin{array}{clr} 
& -\nabla \cdot \mathbf{T}_{1}\left(\mathbf{u}_{1}, p_{1}\right)=\mathbf{f}_{1} & \text { in } \Omega_{1} \\
\text { Stokes system: } & \nabla \cdot \mathbf{u}_{1}=0 & \text { in } \Omega_{1} \\
& \mathbf{u}_{1}=\boldsymbol{\lambda}_{1} & \text { on } \Gamma_{1} \\
& \boldsymbol{\alpha}^{-1} \mathbf{u}_{2}+\nabla p_{2}=\mathbf{f}_{2} & \text { in } \Omega_{2} \\
\text { Darcy system: } & \nabla \cdot \mathbf{u}_{2}=0 & \text { in } \Omega_{2} \\
& p_{2}=\lambda_{2} & \text { on } \Gamma_{2} .
\end{array}
$$

(Both problems must be supplemented with suitable boundary conditions on $\partial \Omega_{i} \backslash \Gamma_{i}$.)

The unknown controls $\lambda_{1}$ and $\lambda_{2}$ can be obtained by minimizing a suitable cost functional that measures the gap between the velocities $\mathbf{u}_{1}=\mathbf{u}_{1}\left(\boldsymbol{\lambda}_{1}\right), \mathbf{u}_{2}=\mathbf{u}_{2}\left(\lambda_{2}\right)$ and the pressures $p_{1}=p_{1}\left(\boldsymbol{\lambda}_{1}\right)$, $p_{2}=p_{2}\left(\lambda_{2}\right)$ on the interfaces $\Gamma_{1}$ and $\Gamma_{2}$, respectively, in a suitable norm. More precisely,

$$
\left(\boldsymbol{\lambda}_{1}, \lambda_{2}\right)=\underset{\left(\boldsymbol{\mu}_{1}, \mu_{2}\right)}{\operatorname{argmin}}\left[J\left(\boldsymbol{\mu}_{1}, \mu_{2}\right)=\frac{1}{2}\left\|\mathbf{u}_{1}\left(\boldsymbol{\mu}_{1}\right)-\mathbf{u}_{2}\left(\mu_{2}\right)\right\|_{\Gamma_{1}}^{2}+\frac{1}{2}\left\|p_{2}\left(\mu_{2}\right)-p_{1}\left(\boldsymbol{\mu}_{1}\right)\right\|_{\Gamma_{2}}^{2}\right] .
$$

Problem (26)-(28) is an optimal control problem in which $\left(\mathbf{u}_{i}, p_{i}\right)(i=1,2)$ represent the state variables. Remark that when $\inf J=0$, the solution of (26)-(28) features the continuity of the velocity field on the interface $\Gamma_{1}$ and the continuity of the pressure on $\Gamma_{2}$.

An $h p$-FEM discretisation using finite dimensional spaces analogous to (15) can be considered also for the Stokes-Darcy problem. In this case the discrete spaces must satisfy the inf-sup condition otherwise suitable stabilization techniques must be adopted [37]. A detailed derivation of the discrete weak formulation of ICDD for the Stokes-Darcy problem is carried out in [8] together with its analysis. In this paper we provide instead an abstract theoretical setting in which both the advection/advection-diffusion and the Stokes-Darcy problems can be cast.

\section{ANALYSIS OF THE ICDD METHOD FOR GENERAL HETEROGENEOUS COUPLINGS}

We rewrite the problems introduced in the previous sections in a general abstract form that is suitable for the analysis of several kinds of heterogeneous coupled problems.

Let us consider an overlapping decomposition of the computational domain $\Omega$ as described in Section 2.1 and the following problem: given $f_{i}$ for $i=1,2$, look for $u_{i}^{\lambda, f}$ s.t.

$$
\begin{aligned}
& L_{i} u_{i}^{\boldsymbol{\lambda}, f}=f_{i} \quad \text { in } \Omega_{i}, \\
& B_{i} u_{i}^{\boldsymbol{\lambda}, f}=0 \quad \text { on } \partial \widetilde{\Omega_{i} \backslash \Gamma_{i}}, \quad \text { for } i=1,2 \text {, } \\
& T_{i} u_{i}^{\lambda, f}=\lambda_{i} \quad \text { on } \widetilde{\Gamma_{i}} \text {, } \\
& \boldsymbol{\lambda}=\left(\lambda_{1}, \lambda_{2}\right)=\underset{\boldsymbol{\mu}=\left(\mu_{1}, \mu_{2}\right)}{\operatorname{argmin}}\left[J(\boldsymbol{\mu})=\frac{1}{2} \sum_{\substack{i=1 \\
(j=3-i)}}^{2}\left\|T_{i} u_{i}^{\boldsymbol{\mu}, f}-\widehat{T}_{i j} u_{j}^{\boldsymbol{\mu}, f}\right\|_{*, \widetilde{\Gamma}_{i}}^{2}\right] \text {, }
\end{aligned}
$$

where:

- $u_{i}=u_{i}^{\boldsymbol{\lambda}, f}$ is the state solution, whose components can be either of the same nature (in the simpler case $u_{i}$ is a scalar variable, otherwise $u_{i}$ collects the components of a vector variable in $\mathbb{R}^{d}$ ), or of different type (like in a saddle point problem). In the latter case we distinguish between the primal variable (e.g. the velocity in the Stokes equations, or the pressure in the Darcy one) and the dual variable (e.g. the pressure in the Stokes equations, or the velocity for Darcy);

- $L_{i}$ is a linear differential operator;

- $B_{i}$ is a suitable linear boundary operator associated with $L_{i}$;

- $T_{i}$ is the zeroth-order trace operator (Dirichlet trace) on $\widetilde{\Gamma}_{i}$ defined on the space of the primal 
variable of $u_{i}$, such that (29) is well posed in $\Omega_{i}$;

- the definition of $\widehat{T}_{i j}$ depends on both operators $L_{1}$ and $L_{2}$ and on the nature of the state variables $u_{1}$ and $u_{2}$. We give here a formal characterization as follows: if $u_{1}$ and $u_{2}$ represent the same kind of variable, then $\widehat{T}_{i j} u_{j}$ is simply the zeroth-order trace on $\widetilde{\Gamma}_{i}$ of $u_{j}$; if the dual variable of $u_{j}$ has the same meaning of the primal variable of $u_{i}$ (e.g. the velocity is the dual variable in the Darcy problem and the primal one for Stokes), then $\widehat{T}_{i j} u_{j}$ is the zeroth-order trace on $\widetilde{\Gamma}_{i}$ of the dual variable of $u_{j}$; - for any $S \subset \partial \Omega_{i}, \widetilde{S} \subseteq S$ is such that the boundary conditions on $\widetilde{S}$ are well-posed for the differential problem (29) (e.g. $\widetilde{S}=S$ for elliptic, Navier-Stokes and Darcy equations, $\widetilde{S}=S^{\text {in }}$ for an advection equation);

- the interface norm in (30) needs to be suitably defined in view of the regularity of the components of $u_{i}$.

\section{Examples:}

1. Advection - Advection/Diffusion equations

$L_{1} u_{1}=-\operatorname{div}\left(\mathbf{b} u_{1}\right)+\gamma u_{1}, B_{1} u_{1}=u_{1}, L_{2} u_{2}=\operatorname{div}\left(-\nu \nabla u_{2}+\mathbf{b} u_{2}\right)+\gamma u_{2}, B_{2} u_{2}=u_{2}$, $\partial \widetilde{\Omega_{i} \backslash \Gamma_{1}}=\left(\partial \Omega_{i} \backslash \Gamma_{1}\right)^{i n}, \widetilde{\Gamma}_{1}=\Gamma_{1}^{i n}, \partial \widetilde{\Omega_{i} \backslash \Gamma_{2}}=\partial \Omega_{2} \backslash \Gamma_{2}, \widetilde{\Gamma}_{2}=\Gamma_{2},\|\cdot\|_{*, \widetilde{\Gamma}_{i}}=\|\cdot\|_{L_{\mathbf{b}}^{2}\left(\widetilde{\Gamma}_{i}\right)}$, $T_{i} u_{i}=\left.u_{i}\right|_{\widetilde{\Gamma}_{i}}, \widehat{T}_{i j} u_{j}=\left.u_{j}\right|_{\widetilde{\Gamma}_{i}}$, for $i=1,2$.

2. Stokes-Darcy equations

$u_{1}=\left(\mathbf{u}_{1}, p_{1}\right), u_{2}=\left(p_{2}, \mathbf{u}_{2}\right)$ (the first component is the primal variable),

$L_{1} u_{1}=\left[\begin{array}{c}-\nabla \cdot \mathbf{T}_{1}\left(\mathbf{u}_{1}, p_{1}\right) \\ \nabla \cdot \mathbf{u}_{1}\end{array}\right], \quad f_{1}=\left[\begin{array}{l}\mathbf{f}_{1} \\ 0\end{array}\right]$,

$B_{1} u_{1}=\mathbf{u}_{1}$ or $B_{1} u_{1}=\mathbf{T}_{1}\left(\mathbf{u}_{1}, p_{1}\right) \cdot \mathbf{n}$,

$L_{2} u_{2}=\left[\begin{array}{c}\boldsymbol{\alpha}^{-1} \mathbf{u}_{2}+\nabla p_{2} \\ \nabla \cdot \mathbf{u}_{2}\end{array}\right], \quad f_{2}=\left[\begin{array}{c}\mathbf{f}_{2} \\ 0\end{array}\right], B_{2} u_{2}=p_{2}$ or $B_{2} u_{2}=\mathbf{u}_{2} \cdot \mathbf{n}$,

$\partial \widetilde{\Omega_{i} \backslash \Gamma_{i}}=\partial \Omega_{i} \backslash \Gamma_{i}, \widetilde{\Gamma}_{i}=\Gamma_{i},\|\cdot\|_{*, \widetilde{\Gamma}_{i}}=\|\cdot\|_{L^{2}\left(\Gamma_{i}\right)}$ for $i=1,2$,

$T_{1} \mathbf{u}_{1}=\mathbf{u}_{1 \mid \Gamma_{1}}, \widehat{T}_{21} p_{1}=p_{1 \mid \Gamma_{2}}, T_{2} p_{2}=p_{2 \mid \Gamma_{2}}, \widehat{T}_{12} \mathbf{u}_{2}=\mathbf{u}_{2 \mid \Gamma_{1}}$.

3. Viscous-inviscid equations for compressible flows (see [2, Sect. 8.4])

$u_{1}=\left(\mathbf{u}_{1}, p_{1}\right), u_{2}=\left(p_{2}, \mathbf{u}_{2}\right)$ (the first component is the primal variable),

$L_{1} u_{1}=\left[\begin{array}{c}\alpha \mathbf{u}_{1}-\nu \Delta \mathbf{u}_{1}-\gamma \nabla\left(\nabla \cdot \mathbf{u}_{1}\right)+\beta \nabla p_{1} \\ \alpha p_{1}+\nabla \cdot \mathbf{u}_{1}\end{array}\right], \quad f_{1}=\left[\begin{array}{l}\mathbf{f}_{1} \\ g_{1}\end{array}\right]$,

$B_{1} u_{1}=\mathbf{u}_{1}$ or $B_{1} u_{1}=\nu\left(\nabla \mathbf{u}_{1}+\left(\nabla \mathbf{u}_{1}\right)^{T}\right) \mathbf{n}-p_{1} \mathbf{n}$,

$L_{2} u_{2}=\left[\begin{array}{c}\alpha \mathbf{u}_{2}+\beta \nabla p_{2} \\ \alpha p_{2}+\nabla \cdot \mathbf{u}_{2}\end{array}\right], \quad f_{2}=\left[\begin{array}{c}\mathbf{f}_{2} \\ g_{2}\end{array}\right], B_{2} u_{2}=p_{2}$ or $B_{2} u_{2}=\mathbf{u}_{2} \cdot \mathbf{n}$,

$\partial \widetilde{\Omega_{i} \backslash \Gamma_{i}}=\partial \Omega_{i} \backslash \Gamma_{i}, \widetilde{\Gamma}_{i}=\Gamma_{i},\|\cdot\|_{*, \widetilde{\Gamma}_{i}}=\|\cdot\|_{L^{2}\left(\Gamma_{i}\right)}$ for $i=1,2$,

$T_{1} \mathbf{u}_{1}=\mathbf{u}_{1 \mid \Gamma_{1}}, \widehat{T}_{21} p_{1}=p_{1 \mid \Gamma_{2}}, T_{2} p_{2}=p_{2 \mid \Gamma_{2}}, \widehat{T}_{12} \mathbf{u}_{2}=\mathbf{u}_{2 \mid \Gamma_{1}}, \nu$ is the kinematic viscosity of the fluid and $\alpha, \beta, \gamma>0$ are given coefficients.

Now we write the discrete weak form of problem (29)- (30). Let $h$ denote a generic discretization parameter, $V_{i, h}$ and $\Lambda_{i, h}$ be finite dimensional spaces approximating the spaces of continuous states and controls, respectively, and let $V_{i, h}^{0}=\left\{v_{i, h} \in V_{i, h}: v_{i, h}=0\right.$ on $\left.\widetilde{\partial \Omega_{i}}\right\}$. Then we introduce two discrete bilinear forms $\mathcal{A}_{i, h}, \mathcal{B}_{i, h}: V_{i, h} \times V_{i, h} \rightarrow \mathbb{R}$, a linear cost functional $\mathcal{F}_{i, h}: V_{i, h} \rightarrow \mathbb{R}$ and a lifting operator $E_{i, h}: \Lambda_{i, h} \rightarrow V_{i, h}$ such that $T_{i}\left(E_{i, h} \lambda_{i, h}\right)=\lambda_{i, h}$.

The discrete weak counterpart of (29)-(30) reads: find $\lambda_{i, h} \in \Lambda_{i, h}, u_{i, h}^{\boldsymbol{\lambda}_{h}, f} \in V_{i, h}$ (for $i=1,2$ ) s.t.

$$
\begin{aligned}
& u_{i, h}^{\boldsymbol{\lambda}_{h}, f}=u_{i, 0, h}+E_{i, h} \lambda_{i, h} \text { where } \\
& \mathcal{A}_{i, h}\left(u_{i, 0, h}, v_{i, h}\right)+\mathcal{B}_{i, h}\left(E_{i, h} \lambda_{i, h}, v_{i, h}\right)=\mathcal{F}_{i, h}\left(v_{i, h}\right) \quad \forall v_{i, h} \in V_{i, h}^{0} \\
& \boldsymbol{\lambda}_{h}=\left(\lambda_{1, h}, \lambda_{2, h}\right)=\underset{\substack{\boldsymbol{\mu}_{h}=\left(\mu_{1, h}, \mu_{2, h}\right) \\
\operatorname{argmin}}}{n}\left[J\left(\boldsymbol{\mu}_{h}\right)=\frac{1}{2} \sum_{\substack{i=1 \\
(j=3-i)}}^{2}\left\|T_{i} u_{i, h}^{\boldsymbol{\mu}_{h}, f}-\widehat{T}_{i j} u_{j, h}^{\boldsymbol{\mu}_{h}, f}\right\|_{*, \widetilde{\Gamma}_{i}}^{2}\right] .
\end{aligned}
$$


When $f=0$, we shorten the notation by writing $u_{i, h}^{\boldsymbol{\lambda}_{h}}=u_{i, h}^{\boldsymbol{\lambda}_{h}, 0}$.

We assume that the discrete spaces $V_{i, h}$ and $\Lambda_{i, h}$ guarantee existence and uniqueness of the solution of $(31)_{1,2}$ as well as its stability. Alternatively, let $\mathcal{A}_{i, h}, \mathcal{B}_{i, h}$ and $\mathcal{F}_{i, h}$ include stabilizing terms.

We analyze the well-posedness of the optimal control problem (31).

Let us define $\boldsymbol{\Lambda}_{h}=\Lambda_{1, h} \times \Lambda_{2, h}$ and

$$
\left\|\boldsymbol{\lambda}_{h}\right\|=\left(\sum_{\substack{i=1 \\(j=3-i)}}^{2}\left\|T_{i} u_{i, h}^{\boldsymbol{\lambda}_{h}}-\widehat{T}_{i j} u_{j, h}^{\boldsymbol{\lambda}_{h}}\right\|_{*, \widetilde{\Gamma}_{i}}^{2}\right)^{1 / 2} \quad \forall \boldsymbol{\lambda}_{h} \in \boldsymbol{\Lambda}_{h} .
$$

The following lemma holds.

\section{Lemma 5.1}

$\|\cdot\|$ is a norm on the control space $\Lambda_{h}$.

Proof. $\|\cdot\|$ is obviously a seminorm on $\boldsymbol{\Lambda}_{h}$, therefore we limit ourselves to show that $\left\|\boldsymbol{\lambda}_{h}\right\|=0$ implies $\boldsymbol{\lambda}_{h}=\mathbf{0}$.

The property $\left\|\boldsymbol{\lambda}_{h}\right\|=0$ holds if and only if the solution of (31) with $f=0$ satisfies $\lambda_{1, h}=$ $T_{1} u_{1, h}^{\boldsymbol{\lambda}_{h}}=\widehat{T}_{12} u_{2, h}^{\boldsymbol{\lambda}_{h}}$ on $\widetilde{\Gamma}_{1}$ and $\lambda_{2, h}=T_{2} u_{2, h}^{\boldsymbol{\lambda}_{h}}=\widehat{T}_{21} u_{1, h}^{\boldsymbol{\lambda}_{h}}$ on $\widetilde{\Gamma}_{2}$, that is, if and only if

$$
\begin{array}{ll}
\mathcal{A}_{1, h}\left(u_{1,0, h}, v_{1, h}\right)+\mathcal{B}_{1, h}\left(E_{1, h} \lambda_{1, h}, v_{1, h}\right)=0 & \forall v_{1, h} \in V_{1, h}^{0} \\
u_{1, h}^{\boldsymbol{\lambda}_{h}}=u_{1,0, h}+E_{1, h} \lambda_{1, h} & \\
\lambda_{1, h}=\widehat{T}_{12} u_{2, h}^{\boldsymbol{\lambda}_{h}} & \\
\mathcal{A}_{2, h}\left(u_{2,0, h}, v_{2, h}\right)+\mathcal{B}_{2, h}\left(E_{2, h} \lambda_{2, h}, v_{2, h}\right)=0 & \forall v_{2, h} \in V_{2, h}^{0} \\
u_{2, h}^{\boldsymbol{\lambda}_{h}}=u_{2,0, h}+E_{2, h} \lambda_{2, h} & \\
\lambda_{2, h}=\widehat{T}_{21} u_{1, h}^{\boldsymbol{\lambda}_{h}} . &
\end{array}
$$

Using standard notations, the algebraic counterpart of (33) can be written as follows

$$
\underbrace{\left[\begin{array}{cc|cc}
A_{11} & A_{1 \Gamma_{1}} & 0 & 0 \\
0 & I & -R_{12} & 0 \\
\hline 0 & 0 & A_{22} & A_{2 \Gamma_{2}} \\
-R_{21} & 0 & 0 & I
\end{array}\right]}_{G}\left[\begin{array}{c}
\underline{\mathbf{U}}_{1} \\
\underline{\boldsymbol{\lambda}}_{1} \\
\hline \underline{\mathbf{U}}_{2} \\
\underline{\boldsymbol{\lambda}}_{2}
\end{array}\right]=\left[\begin{array}{l}
\mathbf{0} \\
\mathbf{0} \\
\mathbf{0} \\
\mathbf{0}
\end{array}\right],
$$

where:

- $\underline{\mathbf{U}}_{i}$ contains the degrees of freedom of $u_{i, 0, h}$;

- $\underline{\boldsymbol{\lambda}}_{i}$ contains the degrees of freedom of $\lambda_{i, h}$;

- $A_{i i}$ is the matrix associated with $\mathcal{A}_{i, h}\left(u_{i, 0, h}, v_{i, h}\right)$;

- $A_{i \Gamma_{i}}$ is the matrix associated with $\mathcal{B}_{i, h}\left(E_{i, h} \lambda_{i, h}, v_{i, h}\right)$;

- $R_{i j}$ are matrices of zeros and ones associated with the operators $\widehat{T}_{i j}$.

We want to prove that $\operatorname{Ker}(G)=\left\{[\mathbf{0}, \mathbf{0}, \mathbf{0}, \mathbf{0}]^{T}\right\}$. For that, we will make use of the relations

$$
\operatorname{Ker}(A)=\left(\operatorname{Im}\left(A^{T}\right)\right)^{\perp}, \quad \operatorname{Ker}\left(\left[\begin{array}{l}
B \\
C
\end{array}\right]\right)=\operatorname{Ker}(B) \cap \operatorname{Ker}(C),
$$

that hold for generic rectangular matrices $A, B$ and $C$, such that $B$ and $C$ have the same number of columns.

Let us split matrix $G$ in $2 \times 2$ blocks $G_{i j}(i, j=1,2)$ of size $N_{i} \times N_{j}$, as shown in (34). 
Under the assumption that local subproblems are well-posed, the blocks $G_{i i}$ are non-singular. Moreover, we have

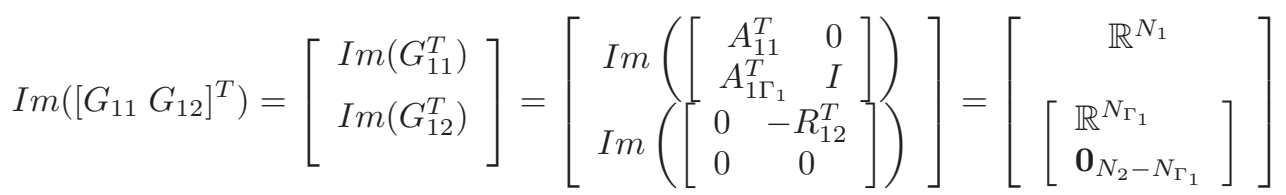

and

$$
\operatorname{Im}\left(\left[G_{21} G_{22}\right]^{T}\right)=\left[\begin{array}{c}
\operatorname{Im}\left(G_{21}^{T}\right) \\
\operatorname{Im}\left(G_{22}^{T}\right)
\end{array}\right]=\left[\begin{array}{c}
\operatorname{Im}\left(\left[\begin{array}{cc}
0 & -R_{21}^{T} \\
0 & 0
\end{array}\right]\right) \\
\operatorname{Im}\left(\left[\begin{array}{cc}
A_{22}^{T} & 0 \\
A_{2 \Gamma_{2}}^{T} & I
\end{array}\right]\right)
\end{array}\right]=\left[\begin{array}{c}
\mathbb{R}^{N_{\Gamma_{2}}} \\
\mathbf{0}_{N_{1}-N_{\Gamma_{2}}}
\end{array}\right] .
$$

Then, thanks to $(35)_{1}$,

$$
\begin{aligned}
& \operatorname{Ker}\left(\left[G_{11} G_{12}\right]\right)=\left(\operatorname{Im}\left(\left[G_{11} G_{12}\right]^{T}\right)\right)^{\perp}=\left[\left[\begin{array}{c}
\mathbf{0}_{N_{1}} \\
\mathbb{R}^{N_{2}-N_{\Gamma_{1}}} \\
\mathbf{0}_{N_{\Gamma_{1}}}
\end{array}\right]\right], \\
& \operatorname{Ker}\left(\left[G_{21} G_{22}\right]\right)=\left(\operatorname{Im}\left(\left[G_{21} G_{22}\right]^{T}\right)\right)^{\perp}=\left[\left[\begin{array}{c}
\mathbb{R}^{N_{1}-N_{\Gamma_{2}}} \\
\mathbf{0}_{N_{\Gamma_{2}}} \\
\mathbf{0}_{N_{2}}
\end{array}\right]\right],
\end{aligned}
$$

and finally, using $(35)_{2}$,

$$
\operatorname{Ker}(G)=\left(\operatorname{Ker}\left(\left[G_{11} G_{12}\right]\right)\right) \cap\left(\operatorname{Ker}\left(\left[G_{21} G_{22}\right]\right)\right)=\left\{[\mathbf{0}, \mathbf{0}, \mathbf{0}, \mathbf{0}]^{T}\right\} .
$$

This implies, in particular, that $\boldsymbol{\lambda}_{1, h}=\mathbf{0}$ and $\lambda_{2, h}=0$. $\square$

Theorem 5.1

The optimal control problem (31) has a unique solution $\boldsymbol{\lambda}_{h} \in \boldsymbol{\Lambda}_{h}$ satisfying the Euler-Lagrange equation

$$
\left\langle J^{\prime}\left(\boldsymbol{\lambda}_{h}\right), \boldsymbol{\eta}_{h}\right\rangle=\sum_{\substack{i=1 \\(j=3-i)}}^{2}\left(T_{i} u_{i, h}^{\boldsymbol{\lambda}_{h}, f}-\widehat{T}_{i j} u_{j, h}^{\boldsymbol{\lambda}_{h}, f}, T_{i} u_{i, h}^{\boldsymbol{\eta}_{h}}-\widehat{T}_{i j} u_{j, h}^{\boldsymbol{\eta}_{h}}\right)_{*, \widetilde{\Gamma}_{i}}=0 \quad \forall \boldsymbol{\eta}_{h} \in \boldsymbol{\Lambda}_{h} .
$$

Proof. By setting

$$
\pi\left(\boldsymbol{\lambda}_{h}, \boldsymbol{\mu}_{h}\right)=\frac{1}{2} \sum_{\substack{i=1 \\(j=3-i)}}^{2}\left(T_{i} u_{i, h}^{\boldsymbol{\lambda}_{h}}-\widehat{T}_{i j} u_{j, h}^{\boldsymbol{\lambda}_{h}}, T_{i} u_{i, h}^{\boldsymbol{\mu}_{h}}-\widehat{T}_{i j} u_{j, h}^{\boldsymbol{\mu}_{h}}\right)_{*, \widetilde{\Gamma}_{i}}
$$

and

$$
\mathcal{L}\left(\boldsymbol{\mu}_{h}\right)=-\frac{1}{2} \sum_{\substack{i=1 \\(j=3-i)}}^{2}\left(T_{i} u_{i, h}^{\mathbf{0}, f}-\widehat{T}_{i j} u_{j, h}^{\mathbf{0}, f}, T_{i} u_{i, h}^{\boldsymbol{\mu}_{h}}-\widehat{T}_{i j} u_{j, h}^{\boldsymbol{\mu}_{h}}\right)_{*, \widetilde{\Gamma}_{i}},
$$

there holds

$$
J\left(\boldsymbol{\mu}_{h}\right)=\pi\left(\boldsymbol{\mu}_{h}, \boldsymbol{\mu}_{h}\right)-2 \mathcal{L}\left(\boldsymbol{\mu}_{h}\right)+\frac{1}{2} \sum_{\substack{i=1 \\(j=3-i)}}^{2}\left\|T_{i} u_{i, h}^{\mathbf{0}, f}-\widehat{T}_{i j} u_{j, h}^{\mathbf{0}, f}\right\|_{*, \widetilde{\Gamma}_{i}}^{2} \cdot
$$

Thanks to Lemma 5.1, the bilinear symmetric form $\pi: \Lambda_{h} \times \Lambda_{h} \rightarrow \mathbb{R}$ is continuous and coercive with respect to the norm $\|\cdot\|$, while $\mathcal{L}: \boldsymbol{\Lambda}_{h} \rightarrow \mathbb{R}$ is a linear and continuous functional. 
Moreover, $\left(\boldsymbol{\Lambda}_{h},\|\cdot\|\right)$ is a finite dimensional Hilbert space and, by applying classical results of calculus of variations, both existence and uniqueness of the solution follow. The Euler-Lagrange equation (36) is obtained by noticing that

$$
\left\langle J^{\prime}\left(\boldsymbol{\lambda}_{h}\right), \boldsymbol{\mu}_{h}\right\rangle=2 \pi\left(\boldsymbol{\lambda}_{h}, \boldsymbol{\mu}_{h}\right)-2 \mathcal{L}\left(\boldsymbol{\mu}_{h}\right) \quad \forall \boldsymbol{\lambda}_{h}, \boldsymbol{\mu}_{h} \in \boldsymbol{\Lambda}_{h} .
$$

\subsection{The optimality system}

We associate the following optimality system with the Euler Lagrange equation (36). Find the state solutions $u_{i, h}=u_{i, h}^{\boldsymbol{\lambda}_{h}, f} \in V_{i, h}$, the dual solutions $w_{i, h} \in V_{i, h}$ and the control $\boldsymbol{\lambda}_{h}=\left(\lambda_{1, h}, \lambda_{2, h}\right) \in$ $\Lambda_{1, h} \times \Lambda_{2, h}$ that satisfy:

$$
\begin{aligned}
& \text { State Problems } \quad(\text { for } i=1,2) \\
& u_{i, h}=u_{i, 0, h}+E_{i, h} \lambda_{i, h}, \text { where } u_{i, 0, h} \in V_{i, h}^{0}: \\
& \mathcal{A}_{i, h}\left(u_{i, 0, h}, v_{i, h}\right)=-\mathcal{B}_{i, h}\left(E_{i, h} \lambda_{i, h}, v_{i, h}\right)+\mathcal{F}_{i, h}\left(v_{i, h}\right) \quad \forall v_{i, h} \in V_{i, h}^{0}
\end{aligned}
$$

Dual Problems (for $i=1,2$ and $j=3-i$ )

$$
\begin{aligned}
& w_{i, h}=w_{i, 0, h}+E_{i, h}\left(T_{i} u_{i, h}-\widehat{T}_{i j} u_{j, h}\right), \text { where } w_{i, 0, h} \in V_{i, h}^{0}: \\
& \mathcal{A}_{i, h}\left(w_{i, 0, h}, v_{i, h}\right)=-\mathcal{B}_{i, h}\left(E_{i, h}\left(T_{i} u_{i, h}-\widehat{T}_{i j} u_{j, h}\right), v_{i, h}\right)+\mathcal{F}_{i, h}\left(v_{i, h}\right) \quad \forall v_{i, h} \in V_{i, h}^{0}
\end{aligned}
$$

Interface Equations $\quad($ for $i=1,2$ and $j=3-i$ )

$$
\left(T_{i} u_{i, h}-\widehat{T}_{i j} u_{j, h}+\widehat{T}_{i j} w_{j, h}, \eta_{i, h}\right)_{*, \widetilde{\Gamma}_{i}}=0 \quad \forall \eta_{i, h} \in \Lambda_{i, h} .
$$

Theorem 5.2

The system (37)-(39) has a unique solution whose control component $\boldsymbol{\lambda}_{h}=\left(\lambda_{1, h}, \lambda_{2, h}\right)$ is the solution of (31) (or equivalently (36), with constraints $\left.(31)_{1,2}\right)$.

Proof. Existence. Let $\boldsymbol{\lambda}_{h}$ be the solution of (31), then (37) are satisfied. Moreover, by choosing $\boldsymbol{\eta}_{h}=\boldsymbol{\lambda}_{h}$ in (36) and thanks to Lemma 5.1, there holds $T_{i} u_{i, h}^{\boldsymbol{\lambda}_{h}, f}-\widehat{T}_{i j} u_{j, h}^{\boldsymbol{\lambda}_{h}, f}=0$ on $\widetilde{\Gamma}_{i}$, thus the solutions $w_{i, h}$ of the dual problems (38) are null in $\bar{\Omega}_{i}$. Therefore, equations (39) are satisfied and (37)-(39) admits at least one solution.

Uniqueness. In view of the linearity of the differential operators $L_{i}$, it suffices to prove that if $f=$ 0 the solution of (37)-(39) is the null one. From (39) it follows that $\widehat{T}_{i j} w_{j, h}=-\left(T_{i} u_{i, h}-\widehat{T}_{i j} u_{j, h}\right)$ on $\widetilde{\Gamma}_{i}$, for $i=1,2$ and $j=3-i$. Therefore, the dual problems (38) become

$$
\begin{array}{ll}
\mathcal{A}_{1, h}\left(w_{1,0, h}, v_{1, h}\right)=-\mathcal{B}_{1, h}\left(E_{1, h}\left(-\widehat{T}_{12} w_{2, h}, v_{1, h}\right)\right. & \forall v_{1, h} \in V_{1, h}^{0} \\
w_{1, h}=w_{1,0, h}+E_{1, h}\left(-\widehat{T}_{12} w_{2, h}\right) & \\
\mathcal{A}_{2, h}\left(w_{2, h}, v_{2, h}\right)=-\mathcal{B}_{2, h}\left(E_{2, h}\left(-\widehat{T}_{21} w_{1, h}\right), v_{2, h}\right) & \forall v_{2, h} \in V_{2, h} \\
w_{2, h}=w_{2,0, h}+E_{2, h}\left(-\widehat{T}_{21} w_{1, h}\right) . &
\end{array}
$$

By the same arguments used in the proof of Lemma 5.1, it follows that $w_{i, h}=0$ in $\bar{\Omega}_{i}$, for $i=1,2$. Then, by (39), we have $T_{1} u_{1, h}=\widehat{T}_{12} u_{2, h}$ on $\widetilde{\Gamma}_{1}$ and $T_{2} u_{2, h}=\widehat{T}_{21} u_{1, h}$ on $\widetilde{\Gamma}_{2}$. By proceeding again as before, it follows $u_{i, h}=0$ in $\bar{\Omega}_{i}$ for $i=1,2$, and then $\lambda_{1, h}=0$ and $\lambda_{2, h}=0$. $\square$

\section{Remark 5.1}

The analysis developed in this Section applies to the A-AD coupling described in Sections 2 and 3 by defining $\mathcal{A}_{i, h}=\mathcal{B}_{i, h}=a_{i, h}$ for $i=1,2$ and following the settings given in Example 1 at the beginning of the present Section. Notice that the interface equation (39) does not yield any information on the control on the part of the interface $\Gamma_{2}$ where $\mathbf{b} \cdot \mathbf{n}=0$, i.e. on $\Gamma_{2}^{0}$. This is the reason why in Section 2 we define the control $\lambda_{2}$ on the subset $\Gamma_{2}^{n z}$, while we impose the natural condition $\nu \partial u_{2} / \partial n_{2}=0$ on $\Gamma_{2}^{0}$. 


\section{Remark 5.2}

Solving the optimality system (37)-(39) is very attractive since both problems with unknowns $u_{i, h}$ and $w_{i, h}$ are of the same nature (no adjoint equations are needed here, contrary to what happens if we integrate by parts following the classical optimal control approaches, as those presented in $[14,15])$.

Moreover, only zeroth-order (Dirichlet) traces are required and no flux has to be computed on the interfaces; the discretizations in $\Omega_{1}$ and $\Omega_{2}$ may be totally unrelated.

If the grids do not match on $\Omega_{12}$, the term $\left(T_{i} u_{i, h}-\widehat{T}_{i j} u_{j, h}\right)$ can be computed through interpolation operators (from the mesh in $\Omega_{1}$ to that in $\Omega_{2}$ or vice versa). When a distributed observation is considered, the interpolation step could be very expensive if the overlapping region is wide and the meshes are very fine in $\Omega_{12}$, unless matching meshes on the overlap are taken into account. Different conclusions can be reached when an interface observation is considered since, in such a case, the interpolation is required only on the interfaces $\widetilde{\Gamma}_{i}$, with a computational cost that does not affect the global efficiency of the method.

\subsection{Algebraic formulation of the optimality system}

In this section we derive the algebraic form of the optimality system, then we propose an efficient algorithm for its solution.

The Optimality System (OS) (37)-(39) has six unknown functions:

- the primal state variables $u_{1, h}$ and $u_{2, h}$,

- the dual state variables $w_{1, h}$ and $w_{2, h}$,

- the control variables $\lambda_{1, h}$ and $\lambda_{2, h}$.

Let us introduce the following arrays and matrices:

$$
\begin{array}{r}
\underline{u}_{1}=\left[u_{1, h}^{0}\left(\mathbf{x}_{j}\right)\right], \quad \underline{w}_{1}=\left[w_{1, h}^{0}\left(\mathbf{x}_{j}\right)\right], \quad \underline{u}_{2}=\left[u_{2, h}^{0}\left(\mathbf{x}_{j}\right)\right], \quad \underline{w}_{2}=\left[w_{2, h}^{0}\left(\mathbf{x}_{j}\right)\right], \\
\underline{\lambda}_{1}=\left[\lambda_{1, h}\left(\mathbf{x}_{j}\right)\right], \text { with } \mathbf{x}_{j} \in \widetilde{\Gamma}_{1}, \quad \underline{\lambda}_{2}=\left[\lambda_{2, h}\left(\mathbf{x}_{j}\right)\right], \text { with } \mathbf{x}_{j} \in \widetilde{\Gamma}_{2}, \\
\mathbf{A}_{i}=\left[\left(a_{i}\right)_{\ell, j}\right]=\left[\mathcal{A}_{i, h}\left(\varphi_{i, j}, \varphi_{i, \ell}\right)\right], \quad \mathbf{B}_{i}=\left[\left(b_{i}\right)_{\ell, j}\right]=\left[\mathcal{B}_{i, h}\left(E_{i, h} \eta_{i, j}, \varphi_{i, \ell}\right)\right], \quad \underline{f}_{i}=\left[\mathcal{F}_{i, h}\left(\varphi_{i, \ell}\right)\right] .
\end{array}
$$

Now, we define:

- $\mathrm{T}_{i j}$, the matrix implementing the interpolation of $\widehat{T}_{i j} u_{j}$ at the nodes of the mesh $\mathcal{M}_{i}$ lying on $\widetilde{\Gamma}_{i}$, - $\mathbf{M}_{i}^{\widetilde{\Gamma}_{i}}$, the $(d-1)$-dimensional mass matrix associated with the interface $\widetilde{\Gamma}_{i}$, and we set

$$
\begin{array}{r}
\mathrm{T}=\left[\begin{array}{ll}
0 & \mathrm{~T}_{12} \\
\mathrm{~T}_{21} & 0
\end{array}\right], \quad \mathrm{A}=\left[\begin{array}{ll}
\mathrm{A}_{1} & 0 \\
0 & \mathrm{~A}_{2}
\end{array}\right], \quad \mathrm{B}=\left[\begin{array}{ll}
\mathrm{B}_{1} & 0 \\
0 & \mathrm{~B}_{2}
\end{array}\right], \quad \mathrm{M}^{\Gamma}=\left[\begin{array}{ll}
\mathrm{M}_{1}^{\widetilde{\Gamma}_{1}} & 0 \\
0 & \mathrm{M}_{2}^{\widetilde{\Gamma}_{2}}
\end{array}\right], \\
\underline{u}=\left[\begin{array}{l}
\underline{u}_{1} \\
\underline{u}_{2}
\end{array}\right], \quad \underline{w}=\left[\begin{array}{l}
\underline{w}_{1} \\
\underline{w}_{2}
\end{array}\right], \quad \underline{\boldsymbol{\lambda}}=\left[\begin{array}{l}
\underline{\lambda}_{1} \\
\underline{\lambda}_{2}
\end{array}\right], \quad \underline{\mathbf{f}}=\left[\begin{array}{l}
\underline{f}_{1} \\
\underline{f}_{2}
\end{array}\right] .
\end{array}
$$

The algebraic counterpart of the OS (37)-(39) reads

$$
\underbrace{\left[\begin{array}{ccc}
\mathrm{A} & 0 & \mathrm{~B} \\
-\mathrm{BT} & \mathrm{A} & \mathrm{B} \\
-\mathrm{M}^{\Gamma} \mathrm{T} & \mathrm{M}^{\Gamma} \mathrm{T} & \mathrm{M}^{\Gamma}
\end{array}\right]}_{\mathrm{G}}\left[\begin{array}{l}
\underline{u} \\
\underline{w} \\
\underline{\underline{\lambda}}
\end{array}\right]=\left[\begin{array}{l}
\mathbf{f} \\
\mathbf{0} \\
\mathbf{0}
\end{array}\right] .
$$

The Schur-complement system associated with (40) reads

$$
\mathrm{S} \underline{\boldsymbol{\lambda}}=\underline{\psi}
$$

where $\underline{\psi}=M^{\Gamma}\left(I-T A^{-1} B\right) T A^{-1} \underline{f}$ and $S$ is the Schur-complement matrix of $G$ with respect to the control variable $\underline{\boldsymbol{\lambda}}$

$$
\mathrm{S}=\mathrm{M}^{\Gamma}\left(\mathrm{I}-\left[\begin{array}{ll}
-\mathrm{T} & \mathrm{T}
\end{array}\right]\left[\begin{array}{cc}
\mathrm{A} & 0 \\
-\mathrm{BT} & \mathrm{A}
\end{array}\right]^{-1}\left[\begin{array}{l}
\mathrm{B} \\
\mathrm{B}
\end{array}\right]\right)=\mathrm{M}^{\Gamma}\left(\mathrm{I}-\left(\mathrm{TA}^{-1} \mathrm{~B}\right)^{2}\right) .
$$


Table I. Summary of equivalent strong-weak-discrete-algebraic formulations of ICDD for heterogeneous couplings

\begin{tabular}{|c|c|}
\hline Continuous strong form of the control problem & $(29)(30)$ \\
\hline Discrete weak form of the control problem & $(31)$ \\
\hline Discrete weak Euler-Lagrange equation & $(36)$ \\
\hline Discrete weak Optimality System (OS) & $(37)(38)(39)$ \\
\hline Algebraic OS & $(40)$ \\
\hline Schur complement form of the OS & $(41)$ \\
\hline
\end{tabular}

Since the mass matrix $\mathrm{M}^{\Gamma}$ is not singular, we can left-multiply the last row of (40) or, equivalently, both sides of (41) by $\left(\mathrm{M}^{\Gamma}\right)^{-1}$. This operation is in fact a preconditioning step of system (41) by the matrix $\mathrm{M}^{\Gamma}$.

The solution of the Schur-complement system (41) can be efficiently computed by Krylov methods as, e.g., Bi-CGStab ([38]). First of all we compute the right-hand side of (41) as described in Algorithm 5.1. Then, given the array $\underline{\boldsymbol{\lambda}}^{(k)}$ at the $k$ th iteration of Bi-CGStab, the matrix-vector product $\underline{\boldsymbol{\chi}}^{(k)}=\mathrm{S} \underline{\boldsymbol{\lambda}}^{(k)}$ is performed by Algorithm 5.2.

Algorithm 5.1 ( $\psi$ evaluation)

Given $\mathbf{f}_{1}$ and $\mathbf{f}_{2}$, compute $\underline{\psi}$.

1. Solve (37) using homogeneous Dirichlet data on the interfaces $\widetilde{\Gamma}_{1}$ and $\widetilde{\Gamma}_{2}$ and right hand sides $\frac{f}{S}$ and $\underline{f}_{2}$, respectively;

2. Solve the dual problems (38);

3. Compute

$$
\underline{\underline{\psi}}^{(k)}=\left[\begin{array}{l}
-\mathrm{T}_{12} \underline{u}_{2}+\mathrm{T}_{12} \underline{w}_{2} \\
\mathrm{~T}_{21} \underline{u}_{1}+\mathrm{T}_{21} \underline{w}_{1}
\end{array}\right] .
$$

Algorithm 5.2 (Schur-complement evaluation)

Given $\underline{\boldsymbol{\lambda}}^{(k)}$, compute $\underline{\boldsymbol{\chi}}^{(k)}=\mathrm{S} \underline{\boldsymbol{\lambda}}^{(k)}$.

1. Solve (37) using $\underline{\lambda}_{1}^{(k)}$ and $\underline{\lambda}_{2}^{(k)}$ as Dirichlet data on the interfaces $\widetilde{\Gamma}_{1}$ and $\widetilde{\Gamma}_{2}$, and null right hand sides $\underline{f}_{1}$ and $\underline{f}_{2}$, respectively;

2. Solve the dual problems (38);

3. Compute

$$
\underline{\boldsymbol{\chi}}^{(k)}=\left[\begin{array}{c}
\underline{\lambda}_{1}^{(k)}-\mathrm{T}_{12} \underline{u}_{2}+\mathrm{T}_{12} \underline{w}_{2} \\
\mathrm{~T}_{21} \underline{u}_{1}-\underline{\lambda}_{2}^{(k)}+\mathrm{T}_{21} \underline{w}_{1}
\end{array}\right] .
$$

We conclude this section by summarizing in Table I all the equivalent formulations of the ICDD method: from the initial continuous strong form of the control problem (29)-(30), to the final Schurcomplement algebraic form (41) implemented in our codes.

\section{NUMERICAL RESULTS FOR A-AD COUPLING}

In this section we report two test cases showing the robustness of the ICDD method with respect to the variations of the viscosity, the overlap thickness and the discretization parameters $h$ and $p$.

We have considered two stopping criteria: one checking the norm of the residual of the linear system (41), i.e.

$$
\frac{\left\|\mathbf{r}^{(k)}\right\|}{\|\boldsymbol{\psi}\|}=\frac{\left\|\boldsymbol{\psi}-\mathbf{S} \boldsymbol{\lambda}^{(k)}\right\|}{\|\boldsymbol{\psi}\|} \leq \epsilon,
$$


the other checking the value of the cost functional $J$, i.e.

$$
J\left(\boldsymbol{\lambda}^{(k)}\right) \leq \epsilon .
$$

The former test is more stringent than the latter, however, if $\epsilon$ is very small, the extra computation required by (43) is not totally justified in the heterogeneous context, as in any case the reduced model we are using in $\Omega_{1}$ represents an approximation of the global elliptic solution.

Even if the ICDD method does not minimize the difference between the hyperbolic solution $u_{1, h}$ and the elliptic one $u_{2, h}$ on the whole overlap, at convergence we measure such a jump by computing the quantity

$$
e_{\Omega_{12}}=\frac{\left\|u_{1, h}-u_{2, h}\right\|_{L^{2}\left(\Omega_{12}\right)}}{\left|\Omega_{12}\right|},
$$

and, although solutions $u_{1, h} \neq u_{2, h}$ do not coincide in the overlapping region, we univocally define the ICDD solution as follows:

$$
u_{I C D D}= \begin{cases}u_{1, h} & \text { in } \Omega_{1} \backslash \Omega_{12} \\ u_{2, h} & \text { in } \Omega_{2} .\end{cases}
$$

We will compare the heterogeneous solution with the numerical solution of the global elliptic problem (2) in $\Omega$, that for better clarity is denoted by $\tilde{u}_{h}$.

The following quantities will be computed to measure the distance between the heterogeneous solution and the global elliptic one:

$$
\tilde{e}_{1}=\frac{\left\|u_{1, h}-\tilde{u}_{h}\right\|_{L^{2}\left(\Omega_{1} \backslash \Omega_{12}\right)}}{\left\|\tilde{u}_{h}\right\|_{L^{2}\left(\Omega_{1} \backslash \Omega_{12}\right)}}, \quad \tilde{e}_{2}=\frac{\left\|u_{2, h}-\tilde{u}_{h}\right\|_{L^{2}\left(\Omega_{2}\right)}}{\left\|\tilde{u}_{h}\right\|_{L^{2}\left(\Omega_{2}\right)}} .
$$

Finally, we will compare the ICDD method with a Sharp Interface (SI) approach based on the transmission conditions (14). The latter is known as the Dirichlet-Neumann method, since in its original version the interface conditions (14) were distributed between the subproblems to provide a Dirichlet condition for the hyperbolic problem and a Neumann condition for the elliptic one. However, since such a method required the tuning of a suitable parameter to accelerate the Richardson iterations, we consider here a more efficient implementation based on solving the associated Steklov-Poincaré equation by the Bi-CGStab method, preconditioned by the SteklovPoincaré operator associated with the elliptic problem in $\Omega_{2}$.

The comparison will highlight both computational cost and accuracy of the solution with respect to the global elliptic solution $\tilde{u}_{h}$.

\subsection{Test case 1}

Let $\Omega=(-1,1)^{2}, \nu$ be a small positive constant that will be specified later, $\mathbf{b}=[y-0.5(x-1), y-$ $0.5(x-1)]^{t}, \gamma=5, f=5.5$.

In order to solve the global homogeneous elliptic problem, we set $u=1$ on the left vertical side of $\Omega$ and $u=0$ on the right one, while on the horizontal side we impose the homogeneous Neumann condition $\partial u / \partial n=0$. When $\nu /|\mathbf{b}|$ is small enough, or equivalently, the Péclet number is large, the solution features a boundary layer on the right side of the domain (in correspondence with the black thick line shown in Fig. 3, left). Thus, it is natural to decompose $\Omega$ in two overlapping subdomains $\Omega_{1}=\left(0, x_{g}+\delta / 2\right) \times(-1,1)$ and $\Omega_{2}=\left(x_{g}-\delta / 2,1\right) \times(-1,1)$ (see Fig. 3), and to solve a reduced advection equation in $\Omega_{1}$ (far from the layer), and the original advection-diffusion equation in $\Omega_{2}$.

Boundary conditions for both the advection and the advection-diffusion subproblems are inherited by the original elliptic problem. More precisely, on $\left(\partial \Omega_{2} \cap \partial \Omega\right)$ and on $\left(\partial \Omega_{1} \cap \partial \Omega\right)^{i n} \subset \partial \Omega_{D}$ we set the same boundary conditions defined above, while no conditions have to be prescribed on $\left(\partial \Omega_{1} \backslash \Gamma_{1}\right)^{\text {out }}$.

We solve the heterogeneous A-AD problem by the ICDD method (5)-(6) for different values of the viscosity $\nu$. Numerical discretization is performed by GLS (Galerkin Least Squares) stabilized $h p$-FEM of $\mathbb{Q}_{p}$ type (see, e.g. [26]). 

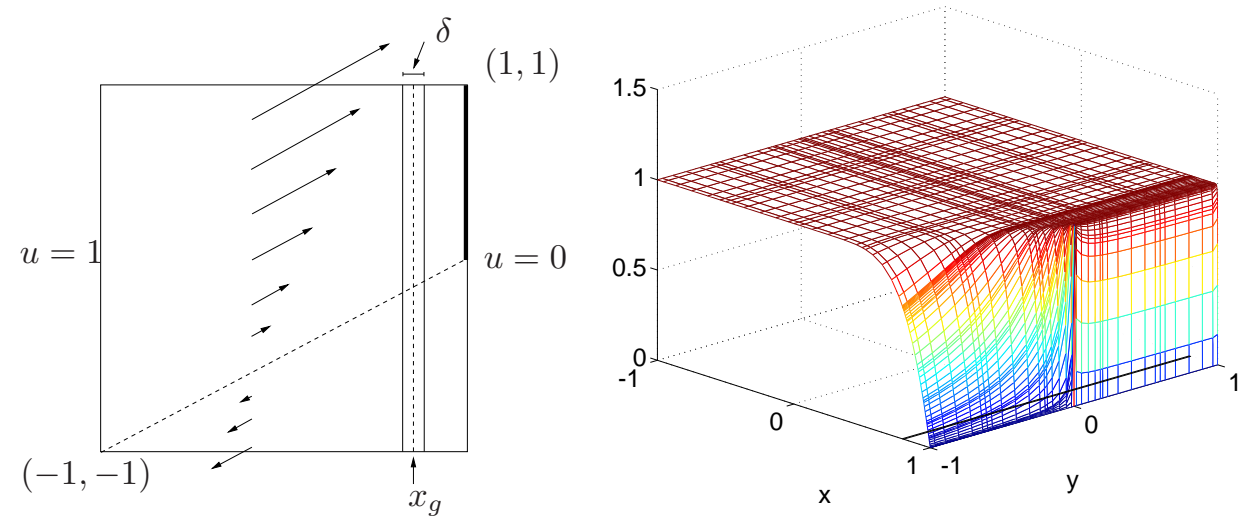

Figure 3. Test 1. On the left, the computational domain, its decomposition in two overlapping subdomains with thickness overlap $\delta$ and the data. On the right, the ICDD solution for $\nu=10^{-8}$ and $\delta=0.01$. The interfaces $\Gamma_{1}$ and $\Gamma_{2}$ are parallel to the $y-$ axis and the corresponding abscissas are $x_{1,2}=0.8 \pm \delta / 2$

Table II. Test 1. ICDD and SI iterations to solve the A-AD coupling, up to tolerance $\epsilon=10^{-9}$. Outside the brackets the iterations to satisfy test (44), inside the brackets those to satisfy (43)

\begin{tabular}{l|ccccc}
\hline$\nu$ & $10^{-4}$ & $10^{-5}$ & $10^{-6}$ & $10^{-7}$ & $10^{-8}$ \\
\hline$\delta$ & \multicolumn{5}{|c}{ ICDD \#it } \\
0.1 & $2(3)$ & $2(3)$ & $2(3)$ & $2(3)$ & $2(3)$ \\
0.01 & $2(4)$ & $2(4)$ & $2(4)$ & $2(4)$ & $2(4)$ \\
0.001 & $3(6)$ & $2(4)$ & $2(3)$ & $2(4)$ & $2(4)$ \\
0.0001 & $4(10)$ & $4(7)$ & $2(4)$ & $2(3)$ & $2(3)$ \\
\hline SI \#it & 2 & 2 & 2 & 2 & 2 \\
\hline
\end{tabular}

In $\Omega_{1}$ we consider a partition of $5 \times 6$ quads, while in $\Omega_{2}$ the partition is made of $8 \times 6$ quads. The use of stabilization techniques on a generic grid does not totally avoid spurious oscillations near the layer, thus the quads are denser towards the right vertical side, where the layer occurs. The polynomial degree is $p=6$ in both subdomains.

The overlap is covered by a strip of flattened quads of thickness $\delta$ along the $x$ direction, while the size of quads along the vertical direction is related to the chosen discretization.

The ICDD solution for $\nu=10^{-8}, x_{g}=0.8$ and $\delta=0.01$ is shown in Figure 3, right.

In Table II we report the number of ICDD iterations needed to satisfy both tests (43) and (44), for several values of both viscosity and $\delta$, when interfaces are located symmetrically around $x_{g}=0.8$. In the same Table we show also the number of Bi-CCStab iterations required by the Sharp Interface (SI) approach to converge, when the interface is located at $x_{g}=0.8$.

We notice that the convergence rate of ICDD is independent of $\delta$ when the viscosity is small, while it mildly depends on $\delta$ for larger values of $\nu$.

In Table III the infimum of the cost functional $J$ attained at convergence for test (44) and the quantities (45) and (46), are shown. Again, $x_{g}=0.8$. Even if ICDD does not enforce any control on the jump between $u_{1, h}$ and $u_{2, h}$ on the whole overlap, the norms $e_{\Omega_{12}}$ turn out to be of the same order of the differences $\tilde{e}_{1}$ and $\tilde{e}_{2}$ between the heterogeneous solution and the original global elliptic one. In the last two columns we report the errors between the heterogeneous and the global elliptic solution for the SI approach. We observe that ICDD and SI provide very similar errors.

We notice that for vanishing $\nu$, the differences $\tilde{e}_{1}$ and $\tilde{e}_{2}$ are bounded from below and the lower bound depends on the position of $x_{g}$. As shown in Table IV, we see that the larger $\Omega_{2}$ the smaller 
Table III. Test 1 . Infimum of the cost functional $J$ when test (43) is used, and differences defined in (45) and (46). $\delta=0.01$, the interfaces $\Gamma_{1}$ and $\Gamma_{2}$ are located at $0.8 \pm \delta / 2$

\begin{tabular}{r|cccc|cc}
\hline \multirow{2}{*}{$\nu$} & \multicolumn{5}{|c|}{ ICDD } & \multicolumn{2}{c}{ Sharp Interface } \\
& $\inf J$ & $e_{\Omega_{12}}$ & $\tilde{e}_{1}$ & $\tilde{e}_{2}$ & $\tilde{e}_{1}$ & $\tilde{e}_{2}$ \\
\hline $10^{-4}$ & $1.19 \mathrm{e}-12$ & $4.66 \mathrm{e}-05$ & $2.20 \mathrm{e}-05$ & $2.77 \mathrm{e}-06$ & $1.86 \mathrm{e}-05$ & $2.26 \mathrm{e}-05$ \\
$10^{-5}$ & $2.11 \mathrm{e}-12$ & $5.03 \mathrm{e}-05$ & $8.74 \mathrm{e}-06$ & $2.82 \mathrm{e}-06$ & $8.38 \mathrm{e}-06$ & $3.32 \mathrm{e}-06$ \\
$10^{-6}$ & $2.06 \mathrm{e}-12$ & $5.05 \mathrm{e}-05$ & $8.72 \mathrm{e}-06$ & $2.84 \mathrm{e}-06$ & $8.29 \mathrm{e}-06$ & $2.38 \mathrm{e}-06$ \\
$10^{-7}$ & $2.05 \mathrm{e}-12$ & $5.05 \mathrm{e}-05$ & $8.74 \mathrm{e}-06$ & $2.84 \mathrm{e}-06$ & $8.29 \mathrm{e}-06$ & $2.36 \mathrm{e}-06$ \\
$10^{-8}$ & $2.04 \mathrm{e}-12$ & $5.05 \mathrm{e}-05$ & $8.75 \mathrm{e}-06$ & $2.84 \mathrm{e}-06$ & $8.29 \mathrm{e}-06$ & $2.36 \mathrm{e}-06$ \\
\hline
\end{tabular}

Table IV. Test 1. Differences (46) between the ICDD heterogeneous and the global elliptic solutions. On the left, for different positions of the interfaces $\Gamma_{1}$ and $\Gamma_{2}$, located at $x_{g} \pm 0.005$. The viscosity is $\nu=10^{-4}$. On the right, versus the overlap thickness $\delta$, when $x_{g}=0.8$ and $\nu=10^{-6}$

\begin{tabular}{c|cc}
\hline$x_{g}$ & $\tilde{e}_{1}$ & $\tilde{e}_{2}$ \\
\hline 0.0 & $1.31 \mathrm{e}-10$ & $3.08 \mathrm{e}-13$ \\
0.2 & $1.94 \mathrm{e}-08$ & $3.81 \mathrm{e}-13$ \\
0.4 & $2.62 \mathrm{e}-07$ & $8.15 \mathrm{e}-13$ \\
0.6 & $3.04 \mathrm{e}-06$ & $3.49 \mathrm{e}-11$ \\
0.8 & $2.20 \mathrm{e}-05$ & $2.77 \mathrm{e}-06$ \\
\hline
\end{tabular}

\begin{tabular}{c|cc}
\hline$\delta$ & $\tilde{e}_{1}$ & $\tilde{e}_{2}$ \\
\hline $10^{-1}$ & $5.65 \mathrm{e}-06$ & $7.38 \mathrm{e}-06$ \\
$10^{-2}$ & $8.72 \mathrm{e}-06$ & $2.84 \mathrm{e}-06$ \\
$10^{-3}$ & $9.94 \mathrm{e}-06$ & $8.74 \mathrm{e}-07$ \\
$10^{-4}$ & $1.01 \mathrm{e}-05$ & $3.13 \mathrm{e}-07$ \\
\hline
\end{tabular}

such differences. As we can see on the right of Table IV, the difference between the heterogeneous and the original elliptic solution is quite independent of the overlap thickness $\delta$.

\subsection{Test case 2}

We consider $\Omega=(-3,7) \times(-3,3) \backslash\left\{(x, y) \in \mathbb{R}^{2}: x^{2}+y^{2}<0.7\right\}$, viscosity $\nu=$ const $>0$ (to be specified later), $\mathbf{b}=[1,0]^{t}, \gamma=1, f=1$, the Dirichlet boundary conditions

$$
u= \begin{cases}1 & \text { on }\{-3\} \times(-3,3) \cup\{7\} \times(-3,3) \\ 0 & \text { on } x^{2}+y^{2}=0.49\end{cases}
$$

and $\mathbf{n} \cdot \nabla u=0$ on the horizontal sides of $\Omega$. When $\nu$ is small with respect to $|\mathbf{b}|$, a boundary layer occurs around the left side of the hole. Then, we decompose the computational domain in 2 overlapping subdomains: the ring $\Omega_{2}=\left\{(x, y) \in \mathbb{R}^{2}: 0.49<x^{2}+y^{2}<2.4025\right\}$ and the domain with hole $\Omega_{1}=(-3,7) \times(-3,3) \backslash\left\{(x, y) \in \mathbb{R}^{2}: x^{2}+y^{2}<2.25\right\}$ (see Fig. 4).

The interfaces $\Gamma_{1}$ and $\Gamma_{2}$ are circles of centre $(0,0)$ and radius $r_{1}=1.55$ and $r_{2}=1.5$, respectively.

Numerical discretization is performed by GLS stabilized $h p$-FEM of $\mathbb{Q}_{p}$ type. The mesh is uniform far from the hole, while inside the square $(-2,2)^{2}$, around the hole, it is finer. The numerical solution of the heterogeneous coupling computed for $\nu=10^{-6}$ is shown in Fig. 4.

In Table $\mathrm{V}$ we show the number of ICDD iterations required to solve the A-AD coupling and to satisfy the stopping test (44) with $\epsilon=10^{-9}$ for different values of the polynomial degree $p$ and for different values of the viscosity. We observe that the convergence rate is independent of both the polynomial degree $p$ and the viscosity. In the same table we report also the number of SI iterations when the sharp interface coincides with $\Gamma_{1}$, i.e. it is a circle of radius $r=1.5$.

In Table VI we show the infimum of the cost functional $J$ attained at convergence for the test (44), as well as the quantities (45) and (46). In the last two columns we report the errors between the heterogeneous and the global elliptic solutions for the SI approach. As in the previous case, 

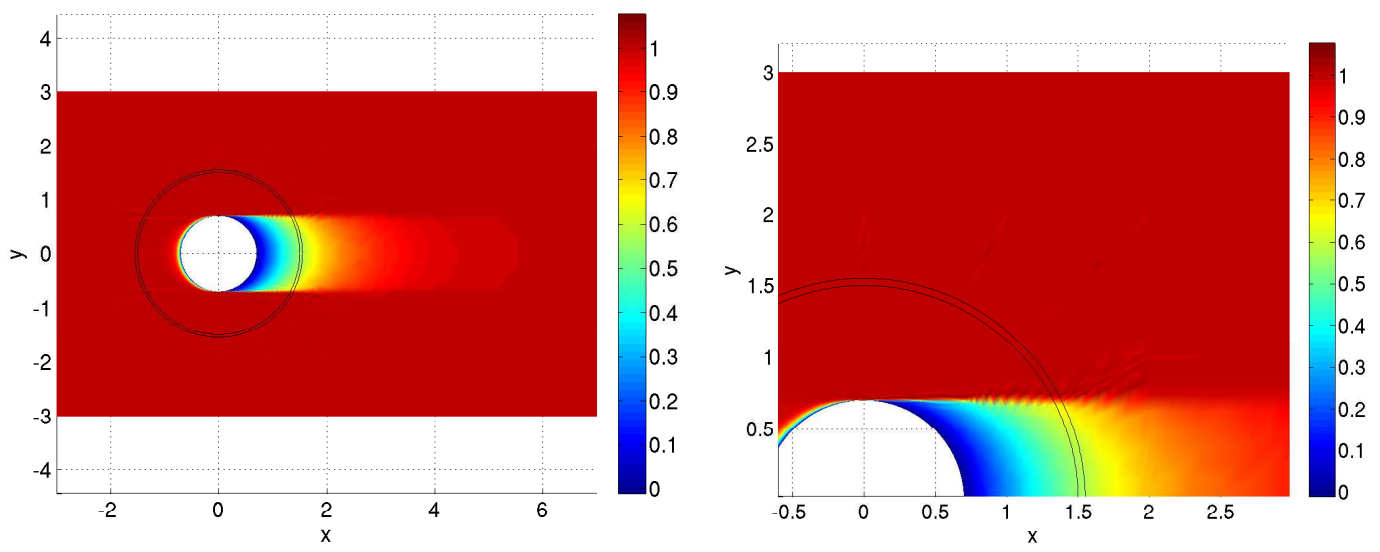

Figure 4. Test 2. ICDD solution of the heterogeneous A-AD coupling for $\nu=10^{-6}$. The thin black lines denote the interfaces. On the right, a zoom of the left picture

Table V. Test 2. ICDD and SI iterations versus the polynomial degree $p$

\begin{tabular}{c|ccc|ccc}
\hline & \multicolumn{3}{|c|}{ ICDD } & \multicolumn{3}{c}{ SI } \\
$\nu$ & $p=4$ & $p=8$ & $p=12$ & $p=4$ & $p=8$ & $p=12$ \\
\hline $10^{-4}$ & 3 & 3 & 3 & 4 & 4 & 4 \\
$10^{-5}$ & 3 & 3 & 3 & 4 & 4 & 4 \\
$10^{-6}$ & 3 & 3 & 3 & 4 & 5 & 5 \\
\hline
\end{tabular}

Table VI. Test 2. Infimum of the cost functional $J$ when test (43) is used, and differences defined in (45) and (46). $\delta=0.05$, the interfaces $\Gamma_{1}$ and $\Gamma_{2}$ are located at $r=1.5$ and $r=1.55$, respectively

\begin{tabular}{r|cccc|cc}
\hline & \multicolumn{5}{|c|}{ ICDD } & \multicolumn{2}{c}{ Sharp Interface } \\
$\nu$ & $\inf J$ & $e_{\Omega_{12}}$ & $\tilde{e}_{1}$ & $\tilde{e}_{2}$ & $\tilde{e}_{1}$ & $\tilde{e}_{2}$ \\
\hline $10^{-4}$ & $9.84 \mathrm{e}-16$ & $7.13 \mathrm{e}-03$ & $1.45 \mathrm{e}-03$ & $1.88 \mathrm{e}-03$ & $1.45 \mathrm{e}-03$ & $1.86 \mathrm{e}-03$ \\
$10^{-5}$ & $7.23 \mathrm{e}-15$ & $1.17 \mathrm{e}-03$ & $1.78 \mathrm{e}-03$ & $2.65 \mathrm{e}-03$ & $1.78 \mathrm{e}-03$ & $2.64 \mathrm{e}-03$ \\
$10^{-6}$ & $9.42 \mathrm{e}-15$ & $6.36 \mathrm{e}-04$ & $1.82 \mathrm{e}-03$ & $2.75 \mathrm{e}-03$ & $1.82 \mathrm{e}-03$ & $2.74 \mathrm{e}-03$ \\
\hline
\end{tabular}

ICDD and SI provide very similar errors.

Computational cost: comparison with the sharp interface approach. One ICDD iteration corresponds to one Bi-CGStab iteration to solve the optimality system (37)-(39). Thus, it requires two matrix-vector products (MVP) and each MVP requires solving two Advection (A) problems and two Advection-Diffusion (AD) problems. We can summarize that the

$$
\text { cost of one ICDD iteration }=(4 \mathrm{~A}+4 \mathrm{AD}) \text { solves. }
$$

Also the Steklov-Poincare equation associated with the sharp interface approach can be solved by a Preconditioned Bi-CGstab method. In this case one MVP costs one Advection plus one Advection-Diffusion problem, while using the preconditioner costs one Advection-Diffusion problem. Therefore, for SI, we have

$$
\text { cost of one PBi-CGstab iteration }=(2 \mathrm{~A}+4 \mathrm{AD}) \text { solves. }
$$


By comparing the number of iterations of Tables II and V and in view of the fact that one ICDD iteration costs a little more than one SI, we conclude that the computational costs of ICDD is only slightly more than that of SI.

Nevertheless, the memory storage required by ICDD is less than the one required by SI. As a matter of fact, in ICDD the matrices of the Advection problem are the same for both primal and dual states (and the same happens for AD), while in the case of SI, the preconditioner does not coincide with the matrix of the primal problem, in view of the different boundary conditions at the interface that characterize the direct local Steklov-Poincaré operator or its inverse (see $[19,21]$ for a more in depth description of this approach).

\section{NUMERICAL RESULTS FOR THE STOKES-DARCY COUPLING}

In [8] the robustness of ICDD versus the discretization parameters ( $h$ and $p$ ) as well as versus the overlap thickness $\delta=\min _{\mathbf{x}_{1} \in \Gamma_{1}, \mathbf{x}_{2} \in \Gamma_{2}} \operatorname{dist}\left(\mathbf{x}_{1}, \mathbf{x}_{2}\right)>0$ has been assessed.

We experienced that the choice of $\delta$ affects the Stokes solution computed by the ICDD method. At the same time it is known (see, e.g., [39, 35, 40]) that an internal layer of thickness $\mathcal{O}(\sqrt{\kappa})=\mathcal{O}(\varepsilon)$ occurs between the fluid and the porous domains. Thus, it seems meaningful to set $\delta=c \sqrt{\kappa}$, where $c>0$ is a suitable constant that may depend on the geometry of the porous medium.

In [8], such a choice of $\delta$ is supported by numerical results showing very high adherence between the ICDD and the SI-BJS solutions (i.e. the solution computed by the Sharp Interface approach with the BJS interface conditions (25)) for both "near parallel flows" (the classical test case of Beavers and Joseph [10], for which the pressure gradient in the Darcy domain is parallel to the interface) and "near normal flows" (in this case the pressure gradient in the Darcy domain is almost normal to the interface).

Moreover, numerical results in [8] highlight that the convergence rate of ICDD is independent of the discretization parameters, and the number of iterations of ICDD is \#it $\simeq \mathcal{O}\left(\delta^{q}\right)$ with $-1 \leq q \leq-1 / 5$ when the permeability $\kappa$ is large, while $\# i t \leq C$ when $\kappa$ is small. However, since $\delta=\sqrt{\kappa}$, the previous estimate is not penalizing, because when $\kappa$ is large, also $\delta$ is large.

In this paper we provide further numerical results in the regime of "near normal flows", the less investigated in literature. According to the classification proposed by Levy and Sanchez-Palencia ([9]), "near normal flows" are characterized by a small projection of $\nabla p_{2}$ on the nominal interface.

\subsection{Test 3: Isotropic non-homogeneous porous media}

This case addresses the Stokes-Darcy coupling with isotropic non-homogeneous porous media and either straight or piecewise linear interface.

The domain is $\Omega=(0,0.1) \times(0,0.1)$ (in meters). First, the nominal interface is set at $z_{\Gamma}=0.08$, in a second time we define a piecewise linear interface $z_{\Gamma}=z_{\Gamma}(x)$ (see Fig. 5). Given the overlap thickness $\delta>0$, the overlapping subdomains are $\Omega_{1}=\left\{(x, z): x \in(0,0.1), z \in\left(z_{\Gamma}(x)-\delta, 0.1\right)\right\}$ and $\Omega_{2}=\left\{(x, z): x \in(0,0.1), z \in\left(0, z_{\Gamma}(x)\right)\right\}$.

The fluid is water with density $\rho=10^{3}\left[\mathrm{~kg} / \mathrm{m}^{3}\right]$ and dynamic viscosity $\mu=10^{-3}[\mathrm{~kg} /(\mathrm{m} \mathrm{s})]$. The heterogeneity of the porous media is measured in terms of its intrinsic permeability by setting

$$
\boldsymbol{\kappa}(x, z)= \begin{cases}10^{-10} \mathbf{I}\left[\mathrm{m}^{2}\right] & 0 \leq x \leq 0.04 \text { and } 0.04 \leq z \leq 0.06 \\ 10^{-14} \mathbf{I}\left[\mathrm{m}^{2}\right] & 0.06 \leq x \leq 0.1 \text { and } 0.02 \leq z \leq 0.04 \\ 10^{-8} \mathbf{I}\left[\mathrm{m}^{2}\right] & \text { elsewhere }\end{cases}
$$

We suppose that the fluid is subject to the gravitational force, thus $\mathbf{f}_{1}=\mathbf{f}_{2}=(0,-\rho g)^{t}\left[\mathrm{~kg} /\left(\mathrm{m}^{2} \mathrm{~s}^{2}\right)\right]$, where $g$ is the gravitational acceleration. The fluid enters into the domain $\Omega_{1}$ through the vertical boundaries. In Figure 5 the hydrodynamic pressure

$$
\tilde{p}_{2}=p_{2}+\rho g\left(z-z_{0}\right)
$$



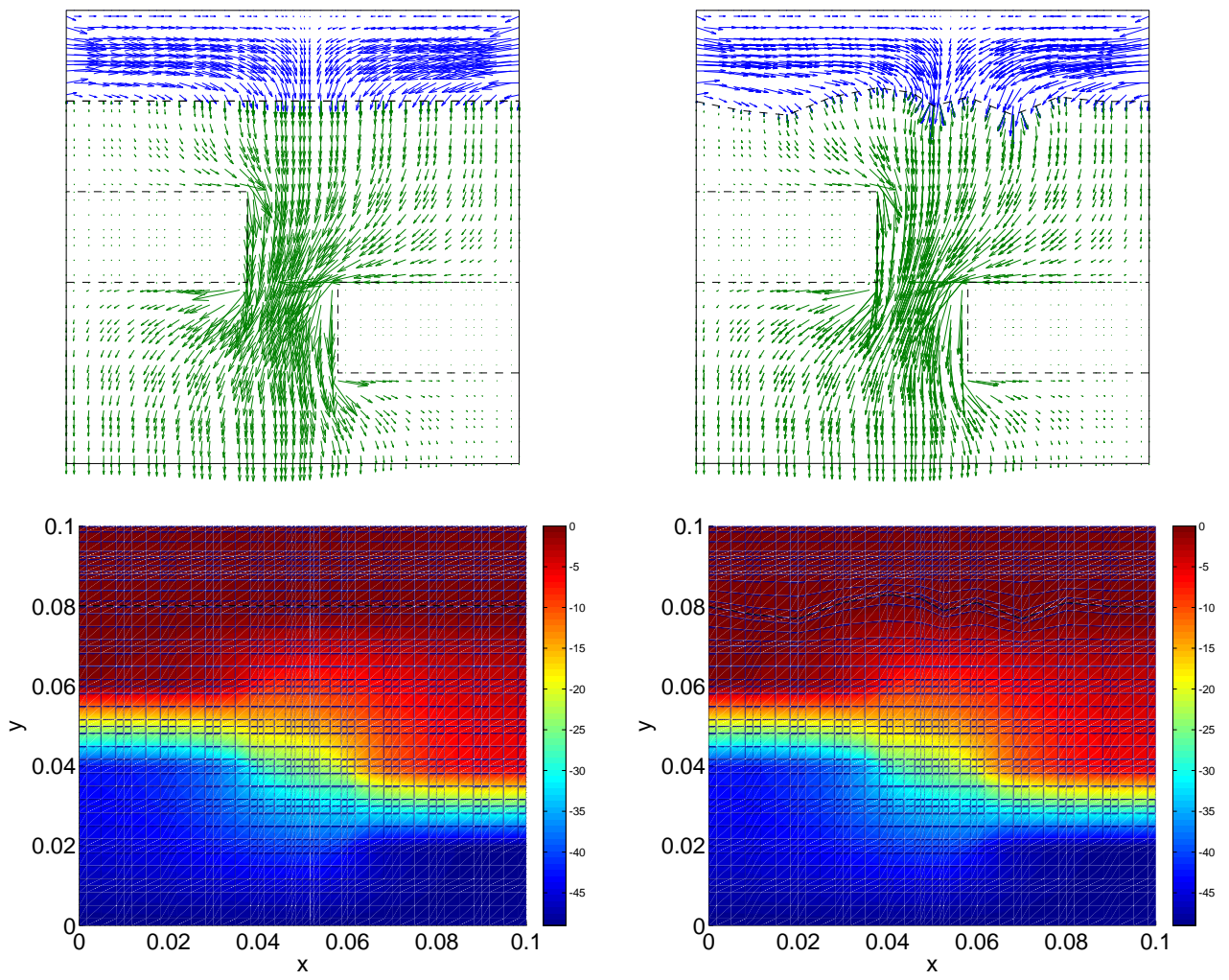

Figure 5. Test 3. The ICDD solution for $\kappa=10^{-8} \mathrm{~m}^{2}$. On the left with straight interface, on the right with a piecewise linear interface

with $z_{0}=0.1$ and the velocity field are shown: on the left with a straight interface, on the right with a piecewise linear interface. In both cases we set $\delta=10^{-4}$ corresponding to $\delta=\sqrt{\kappa}$ with $\kappa=10^{-8}$, that is the permeability in the region close to the interface.

The ICDD solution is computed by discretization with stabilized $\mathbb{Q}_{4}-\mathbb{Q}_{4} h p$-FEM. Convergence of ICDD up to tolerance $\epsilon=10^{-9}$ is achieved in 3 iterations for the straight interface and in 6 iterations for the piecewise linear interface.

Simplicity in handling Dirichlet interface conditions is a strong point of ICDD, whereas the interface conditions of SI-BJS involve both normal and tangential derivatives and they must be accurately set up to take into account possible corners as well as non-straight interfaces.

\subsection{Test 4: Large pressure gradient in the porous domain}

The aim of this test is to investigate the behavior of the velocity around the interface when both components of the pressure gradient inside the porous domain are larger than the corresponding ones in the fluid domain, though keeping valid the assumption that the Reynolds number in $\Omega_{2}$ is small in order to justify the adoption of the Darcy equation.

A coarse analysis leads us to guess that the first component of the velocity is larger in the porous domain than in the fluid one, but it is not evident what happens around the transition region.

At the same time we aim at analyzing the solutions yielded by the SI-BJS and the ICDD approaches. It is well known that the BJS condition (25) 3 was proposed and analyzed (see, e.g. $[11,33])$ in the case of "near parallel flows", i.e. when $\nabla p_{2} \cdot \mathbf{n}_{\Gamma}$ is very small or null.

In this test case we have large values of $\nabla p_{2} \cdot \mathbf{n}_{\Gamma}$, thus we are interested in investigating if (25) 3 keeps holding, as well as in comparing the ICDD and the BJS solutions. 

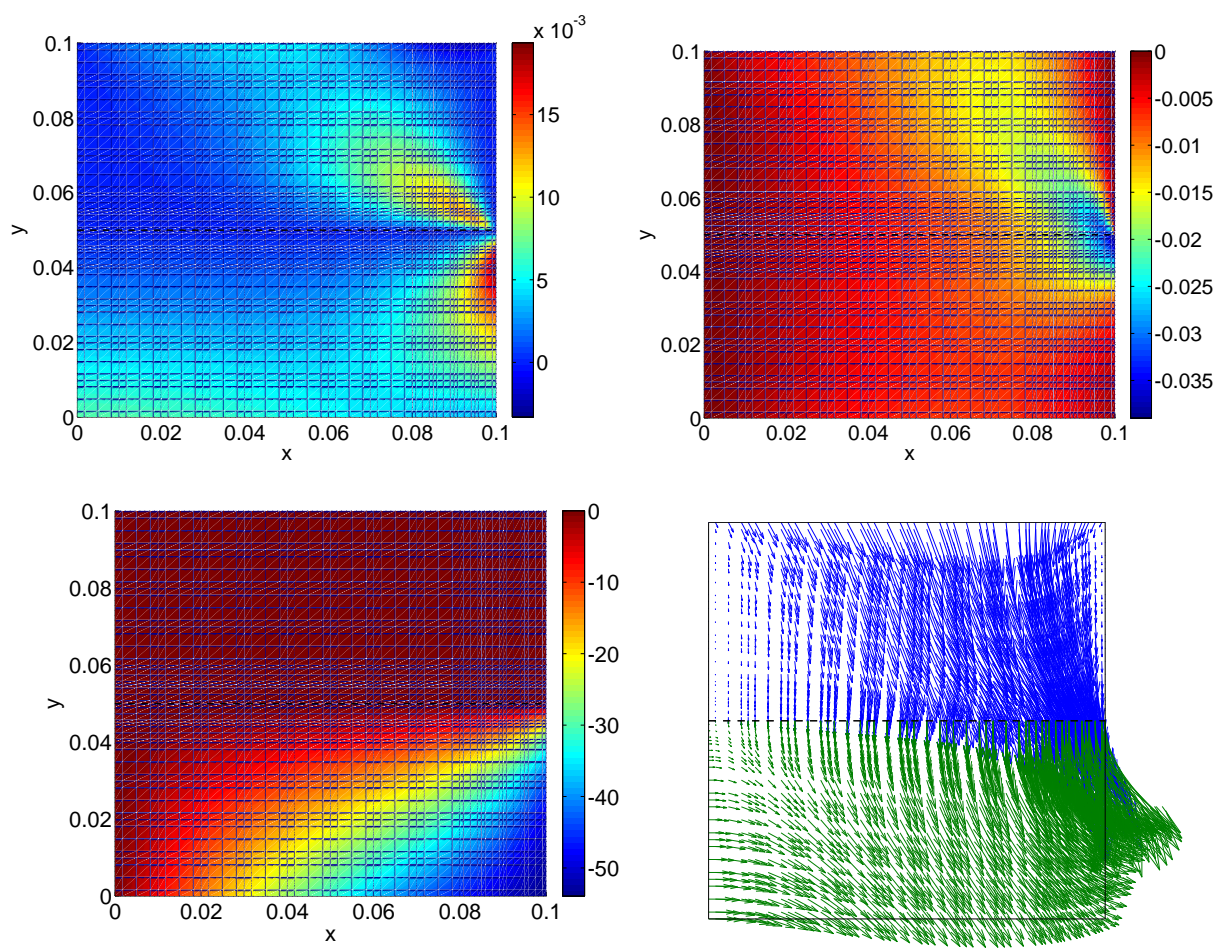

Figure 6. Test 4. Velocity field (first component at top left, second component at top right), pressure (bottom left) and velocity field (bottom right) computed by ICDD with $\delta=10^{-4}$. Discretization by stabilized $\mathbb{Q}_{4}-\mathbb{Q}_{4}$ in both Stokes and Darcy domains. The permeability is $\kappa=\kappa \mathbf{I}$ with $\kappa=10^{-8}$

The domain is $\Omega=(0,0.1) \times(0,0.1)$ (in meters) and the nominal interface is set at $z_{\Gamma}=0.05$. Given the overlap thickness $\delta>0$, the overlapping subdomains are $\Omega_{1}=(0,0.1) \times\left(z_{\Gamma}-\delta, 0.1\right)$ and $\Omega_{2}=(0,0.1) \times\left(0, z_{\Gamma}\right)$.

The fluid is water as in the previous case and we consider homogenenous and isotropic porous media with permeability $\kappa=\kappa \mathbf{I}$.

The fluid is subject to the gravitational force $\mathbf{f}_{1}=\mathbf{f}_{2}=(0,-\rho g)^{T}$. The water enters into the domain $\Omega_{1}$ through the top horizontal side, where we impose $\mathbf{T}\left(\mathbf{u}_{1}, p_{1}\right) \mathbf{n}_{1}=g \rho(z-0.1) \mathbf{n}_{1}$. On the vertical sides of $\Omega_{1}$ we set no-slip boundary conditions.

On the external boundary $\partial \Omega_{2} \cap \partial \Omega$ we set the pressure $p_{2}(x, z)=\rho g(z-0.1)-$ $0.05 \rho \arctan (10 x) \arctan (100(0.05-z))$, so that both vertical and horizontal components of the pressure gradient are large inside the porous domain.

In Fig. 6 we show the hydrodynamic pressure (47) with $z_{0}=0.1$ and the velocity field computed by ICDD method for $\kappa=10^{-8}$ and $\delta=10^{-4}$.

The adherence between the ICDD and the SI-BJS solutions is very high, as we can see from Fig. 7 where we report the profiles of the solutions at three different abscissas $\bar{x} \in\{0.03,0.08,0.0975\}$ when $\kappa=10^{-8}$. For smaller values of $\kappa$, the behaviour of the solutions is similar and it scales as $\kappa$.

Numerical results show that, even if the first component of the velocity reaches large and comparable values inside the Stokes and Darcy domains far from the interface, it is very small in a thin region around the interface and moreover

$$
u_{1}=\mathcal{O}\left(\kappa^{3 / 2}\right), \quad u_{2}=\mathcal{O}\left(\kappa^{2}\right), \quad v_{1}=v_{2}=\mathcal{O}(\kappa), \quad \kappa \rightarrow 0,
$$

being $u_{1}, u_{2}$ and $v_{1}, v_{2}$ the components of the velocity inside the Stokes and the Darcy domains, respectively. We conclude that the Darcy tangential velocity is anyway smaller than the Stokes one around the transition region. 

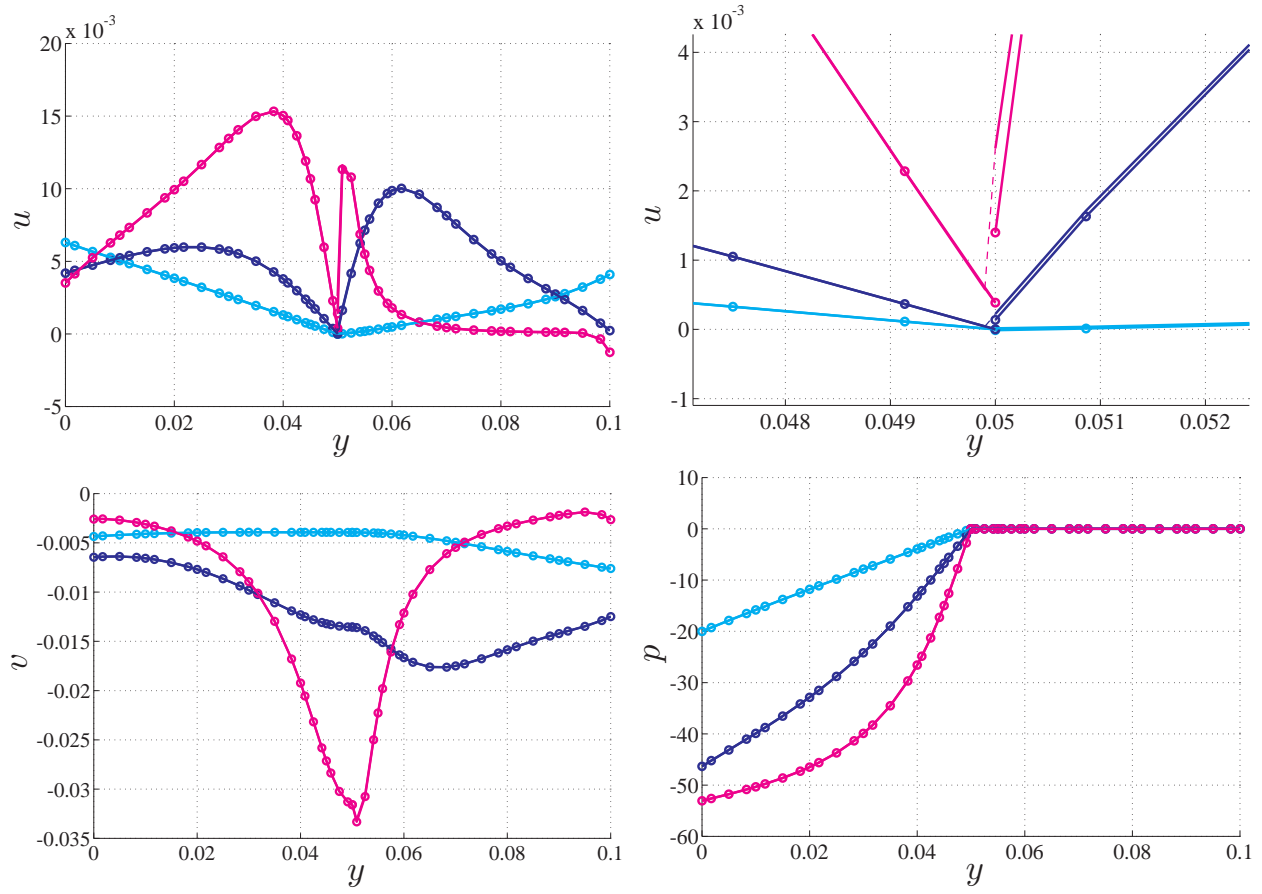

Figure 7. Test 4. At the top, profiles of the first component of the velocity (the picture on the right is a zoom of the left one); at bottom left, profiles of the second component of the velocity; at bottom right, profiles of the hydrodynamic pressure (47). SI-BJS solution (circles) and ICDD solution (continuous lines) at $\bar{x}=0.03$ (light blue), $\bar{x}=0.08$ (dark blue), $\bar{x}=0.0975$ (magenta), when $\kappa=10^{-8}$. The dashed lines in the top-right picture refer to the Stokes solution inside the overlap

We remark that both the SI-BJS and the ICDD approaches yield very similar solutions, although they are grounded in very different bases, and this fact corroborates both methods.

Numerical results show that the ICDD method turns out to be a valid and competitive (in terms of simplicity and computational costs) alternative to the SI-BJS approach. As a matter of fact, the number of iterations required by both ICDD and SI-BJS (preconditioned by the local Darcy Steklov-Poincaré operator) to converge up to a tolerance $\epsilon=10^{-9}$ are 5 and 3 respectively, for all the considered values of $\kappa$. The ICDD computational cost is comparable with that of SI-BJS, provided that the latter is preconditioned in an optimal way (using either the local Stokes or Darcy Steklov-Poincaré operator [28]). On the contrary, ICDD does not require any preconditioner to be efficient. We refer to [8] for a more in-depth comparison of their computational costs.

\section{ACKNOWLEDGEMENT}

The first author acknowledges funding from the European Union Seventh Framework Programme (FP7/2007-2013) under grant agreement n 294229.

\section{REFERENCES}

1. B. Smith, P. Bjørstad, and W. Gropp, Domain Decomposition. Parallel Multilevel Methods for Elliptic Partial Differential Equations. Cambridge: Cambridge University Press, 1996.

2. A. Quarteroni and A. Valli, Domain Decomposition Methods for Partial Differential Equations. Oxford Science Publications, 1999.

3. A. Toselli and O. Widlund, Domain decomposition methods-algorithms and theory, vol. 34 of Springer Series in Computational Mathematics. Berlin: Springer-Verlag, 2005.

4. M. Discacciati, P. Gervasio, and A. Quarteroni, "The interface control domain decomposition (ICDD) method for elliptic problems," SIAM J. Control Optim., vol. 51, no. 5, pp. 3434-3458, 2013. 
5. M. Discacciati, P. Gervasio, and A. Quarteroni, "The interface control domain decomposition (ICDD) method for the Stokes problem," J. Coupled Syst. Multiscale Dyn., vol. 1, no. 5, pp. 372-392, 2013.

6. E. Miglio, A. Quarteroni, and F. Saleri, "Coupling of free surface and groundwater flows," Computers \& fluids, vol. 32, pp. 73-83, 2003.

7. M. Discacciati, E. Miglio, and A. Quarteroni, "Mathematical and numerical models for coupling surface and groundwater flows," Appl. Numer. Math., vol. 43, no. 1-2, pp. 57-74, 2002.

8. M. Discacciati, P. Gervasio, and A. Quarteroni, "Interface Control Domain Decomposition (ICDD) Method for Stokes-Darcy coupling," tech. rep., 17/2014, MOX - Politecnico di Milano, 2014. Submitted.

9. T. Levy and E. Sánchez-Palencia, "On boundary conditions for fluid flow in porous media," Internat. J. Engrg. Sci., vol. 13, no. 11, pp. 923-940, 1975.

10. G. Beavers and D. Joseph, "Boundary conditions at a naturally permeable wall," Numer. Math., vol. 84, no. 2, pp. 173-197, 1999.

11. P. Saffman, "On the boundary condition at the interface of a porous medium," Stud. Appl. Math., vol. 1, pp. 93-101, 1971.

12. P. Grisvard, Elliptic Problems in Nonsmooth Domains. Boston, MA: Pitman (Advanced Publishing Program), 1985.

13. J.-L. Lions, Optimal Control of Systems Governed by Partial Differential Equations. New York: Springer-Verlag, 1971.

14. R. Glowinski, Q. Dinh, and J. Periaux, "Domain decomposition methods for nonlinear problems in fluid dynamics," Comput. Methods Appl. Mech. Engrg., vol. 40, no. 1, pp. 27-109, 1983.

15. J.-L. Lions and O. Pironneau, "Algorithmes parallèles pour la solution de problèmes aux limites," C. R. Acad. Sci. Paris Sér. I Math., vol. t. 327, pp. 947-952, 1998.

16. P. Gervasio, J.-L. Lions, and A. Quarteroni, "Heterogeneous coupling by virtual control methods," Numerische Mathematik, vol. 90, no. 2, pp. 241-264, 2001.

17. V. Agoshkov, P. Gervasio, and A. Quarteroni, "Optimal control in heterogeneous domain decomposition methods for advection-diffusion equations," Mediterr. J. Math., vol. 3, no. 2, pp. 147-176, 2006.

18. M. Discacciati, P. Gervasio, and A. Quarteroni, Heterogeneous mathematical models in fluid dynamics and associated solution algorithms, vol. 2040 of Lecture Notes in Mathematics, ch. 2, pp. 57-123. Springer, 2011. Lectures given at the C.I.M.E. Summer School held in Cetraro, July 2009. Edited by G. Naldi and G. Russo.

19. F. Gastaldi, A. Quarteroni, and G. Sacchi Landriani, "On the coupling of two dimensional hyperbolic and elliptic equations: analytical and numerical approach," in Third International Symposium on Domain Decomposition Methods for Partial Differential Equations (J. T.F.Chan, R.Glowinski and O.B.Widlund, eds.), (Philadelphia), pp. 22-63, SIAM, 1990.

20. P. Blanco, P. Gervasio, and A. Quarteroni, "Extended variational formulation for heterogeneous partial differential equations," Comput. Methods in Applied Math., vol. 11, no. 2, pp. 141-172, 2011.

21. F. Gastaldi and A. Quarteroni, "On the coupling of hyperbolic and parabolic systems: analytical and numerical approach.," Appl. Numer. Math., vol. 6, no. 1, pp. 3-31, 1989.

22. L. Marini and A. Quarteroni, "A relaxation procedure for domain decomposition methods using finite elements," Numer. Math., vol. 55, pp. 575-598, 1989.

23. P. Blanco, P. Gervasio, and A. Quarteroni, "Mortar coupling for heterogeneous partial differential equations," in Domain Decomposition Methods in Science and Engineering XX (DD20, San Diego 2011) (R. Bank, M. Holst, O. Widlund, and J. Xu, eds.), (Berlin), Springer Verlag, 2013.

24. C. Canuto, M. Y. Hussaini, A. Quarteroni, and T. A. Zang, Spectral Methods. Fundamentals in Single Domains. Heidelberg: Springer, 2006.

25. C. Canuto, M. Y. Hussaini, A. Quarteroni, and T. A. Zang, Spectral Methods. Evolution to Complex Geometries and Applications to Fluid Dynamics. Heidelberg: Springer, 2007.

26. A. Quarteroni and A. Valli, Numerical Approximation of Partial Differential Equations. Heidelberg: Springer Verlag, 1994.

27. C. Canuto, P. Gervasio, and A. Quarteroni, "Finite-Element Preconditioning of G-NI Spectral Methods," SIAM J. Sci. Comput., vol. 31, no. 6, pp. 4422-4451, 2009/10.

28. M. Discacciati and A. Quarteroni, "Navier-Stokes/Darcy coupling: modeling, analysis, and numerical approximation," Rev. Mat. Complut., vol. 22, no. 2, pp. 315-426, 2009.

29. J. Bear, Dynamics of Fluids in Porous Media. New York: Dover Publications, Inc., 1972.

30. M. Discacciati, Domain Decomposition Methods for the Coupling of Surface and Groundwater Flows. PhD thesis, Ecole Polytechnique Fédérale de Lausanne, Lausanne (CH), 2004.

31. V. Girault and B. Rivière, "DG approximation of coupled Navier-Stokes and Darcy equations by Beaver-JosephSaffman interface condition," SIAM J. Numer. Anal., vol. 47, no. 3, pp. 2052-2089, 2009.

32. W. Layton, F. Schieweck, and I. Yotov, "Coupling fluid flow with porous media flow," SIAM J. Numer. Anal., vol. 40, no. 6, pp. 2195-2218 (2003), 2002.

33. W. Jäger and A. Mikelić, "On the boundary conditions at the contact interface between a porous medium and a free fluid," Ann. Scuola Norm. Sup. Pisa Cl. Sci. (4), vol. 23, no. 3, pp. 403-465, 1996.

34. B. Rivière and I. Yotov, "Locally conservative coupling of Stokes and Darcy flows," SIAM J. Numer. Anal., vol. 42, no. 5, pp. 1959-1977, 2005.

35. A. Ochoa-Tapia and S. Whitaker, "Momentum transfer at the boundary between a porous medium and a homogeneous fluid I. Theoretical development," Int. J. Heat Mass Transfer, vol. 38, pp. 2635-2646, 1995.

36. A. Ochoa-Tapia and S. Whitaker, "Momentum transfer at the boundary between a porous medium and a homogeneous fluid II. Comparison with experiment," Int. J. Heat Mass Transfer, vol. 38, pp. 2647-2655, 1995.

37. V. Girault and P. Raviart, Finite Element Methods for Navier-Stokes Equations. Theory and Algorithms. Berlin: Springer, 1986.

38. H. van der Vorst, Iterative Krylov methods for large linear systems, vol. 13 of Cambridge Monographs on Applied and Computational Mathematics. Cambridge: Cambridge University Press, 2003. 
39. P. Saffman, "On the boundary condition at the interface of a porous medium," Stud. Appl. Math., vol. 1, pp. 93-101, 1971.

40. W. Jäger and A. Mikelić, "On the interface boundary condition of Beavers, Joseph and Saffman," SIAM J. Appl. Math., vol. 60, no. 4, pp. 1111-1127, 2000. 